\title{
Parasites of marine, freshwater and farmed fishes of Portugal: a review
}

\author{
Parasitos de peixes marinhos, de água doce e de criação de Portugal: uma revisão \\ Jorge da Costa Eiras ${ }^{1 *}$ \\ ${ }^{1}$ Departamento de Biologia, Faculdade de Ciências, Universidade do Porto - UP, Porto, Portugal
}

Received May 16, 2016

Accepted July 20, 2016

\begin{abstract}
An extensive literature review is made of the parasites in marine and freshwater fish in mainland Portugal, the Portuguese archipelagos of the Azores and Madeira, as well as in farmed fish. The host(s) of each parasite species, its location in the host, site of capture of the host, whenever possible, and all the available bibliographic references are described. The economic importance of some parasites and the zoonotic relevance of some parasitic forms are discussed. A general overview of the data is provided, and some research lines are suggested in order to increase and complement the current body of knowledge about the parasites of fish from Portugal.
\end{abstract}

Keywords: Parasites, fish, pathology, economy, zoonotic diseases, Portugal.

\section{Resumo}

É feita uma revisão do conhecimento dos parasitas de peixes de Portugal com base em extensa compilação bibliográfica. São referidos os parasitas de peixes marinhos e de água doce do continente, dos Arquipélagos da Madeira e dos Açores, bem como os parasitas de peixes de piscicultura. Para cada espécie é indicado o hospedeiro, localização no hospedeiro, local de pesca quando possível, e referências bibliográficas disponíveis. Discute-se a importância econômica de alguns dos parasitas e a relevância zoonótica de algumas formas. É feita uma avaliação geral dos dados e sugerem-se linhas de pesquisa que aumentem e complementem o conhecimento atual sobre os parasitas de peixes de Portugal.

Palavras-chave: Parasitas, peixes, patologia, economia, zoonoses, Portugal.

\section{Introduction}

Portugal is a coastal nation in southwestern Europe, which covers an area of about $92,000 \mathrm{~km}^{2}$ and includes the archipelagos of the Azores and Madeira in the Atlantic Ocean. It has a coastline of $943 \mathrm{~km}$ on the mainland, $250 \mathrm{~km}$ on Madeira and $667 \mathrm{~km}$ on Azores. Portugal has the third largest Exclusive Economic Zone (EEZ) in the European Union and the twentieth largest EEZ in the world, with a total of 1,727,408 $\mathrm{km}^{2}$ divided between mainland Portugal $\left(327,667 \mathrm{~km}^{2}\right)$, the Archipelago of Azores $\left(953,633 \mathrm{~km}^{2}\right)$ and the Archipelago of Madeira $\left(446,108 \mathrm{~km}^{2}\right)$ (CARNEIRO et al., 2014). This area will probably increase significantly with new international regulations concerning the limits of the EEZs.

Portugal's marine waters are inhabited by a variety of fish whose exact extent is difficult to determine. For a comprehensive historical background on the knowledge and quantification of species, see Carneiro et al. (2014). According to these authors,

*Corresponding author: Jorge da Costa Eiras. Departamento de Biologia, Faculdade de Ciências, Universidade do Porto, Rua do Campo Alegre, Edifício FC4, 4169-007 Porto, Portugal; CIIMAR (Centro Interdisciplinar de Investigação Marinha e Ambiental), Porto, Portugal. e-mail: jceiras@fc.up.pt there are a total of 1191 different fish species, 734 off mainland Portugal, 857 off Açores and 766 off Madeira. According to Costa FO et al. (2012), based on the FishBase occurrence records, there are 828 different fish species in the three zones. This means that Portugal's marine icthyofauna is probably one of the most diversified in European waters, and explains the high per capita fish consumption in the country, making it the European Union country with the highest average annual per capita consumption of seafood, about $56 \mathrm{~kg}$, which corresponds to $160 \mathrm{~g}$ of seafood per day (CARDOSO et al., 2016). As for freshwater fish, there are 36 known species in mainland Portugal (ALMAÇA, 1996), but we have found no report about freshwater fish in Madeira and Azores.

Research on fish biology in Portugal was developed mostly in the early $20^{\text {th }}$ century. To the best of our knowledge, the first researcher who studied fish parasites was Tendeiro (1955). Other researchers worked in the field, studying mainly marine fish, but for decades their research was very fragmentary and sporadic, and the resulting papers constitute a collection of different observations of several fish species that provide only elementary data on the occurrence 
of some parasites. Important questions such as the host-parasite relationship, parasite biology, pathological consequences of the infections, economic significance of the parasites, etc., were not studied, and for a long time research on fish parasites resulted in no more than a simple list of species infecting a small number of hosts.

This situation improved slightly with the studies of Carvalho Varela et al. (1981), Carvalho Varela (1975, 2005), Carvalho Varela \& Cunha-Ferreira (1984, 1987), Machado Cruz (1959a, b), Menezes (1984, 1992, 1994, 2000), Menezes \& Ré (1991) and Menezes et al. (1990a, b) (see list of References) in the 1970s, which marked the beginning of a new period of fish parasitological studies in Portugal. However, an integrated approach to fish parasitism was still lacking, and more consistent research by a group of young researchers began in the 1980s, most at them affiliated to universities. These researchers applied for national and international funding to support their projects, established fruitful relationships with colleagues at well known international research centers, engaged in cooperative research with some of them, and as part of their academic duties, invested strongly in the supervision of MSc and $\mathrm{PhD}$ students, which led to the production of a number of Dissertations and Thesis on fish parasitology. These efforts resulted in a significant increase in the body of knowledge about fish parasitology in Portugal, and a large increase in the number of qualified researchers. Consequently, numeous papers have been published in important international scientific journals and it can be stated that, today, the scientific community dealing with this field recognizes the studies performed in Portugal and the scientific collaboration developed with foreign researchers. For these reasons, it is important to name the main researchers that contributed significantly to the body of knowledge about fish parasites in the country and invested in the supervising of students who are well known in the field today: in alphabetical order, A Saraiva, C Azevedo, C Cruz, G Costa, JC Eiras and MJ Santos. PhD studies in this field were completed by CJ Francisco, F Cavaleiro, G Casal, HN Cabral, JF Marques, LJ Rangel, $\mathrm{M}$ Hermida and S Rocha. Their work resulted in a comprehensive body of research into the life cycle of parasites, descriptions of new species including their molecular characterization, parasites as biological markers, host-parasite relationships, life cycles, parasites in farmed fish, parasites as human pathogens, ultrastructure of parasites, the economic impact of parasites, histopathological studies, geographical distribution of parasites, etc.

Several papers on general aspects of fish parasitology in Portugal were produced by Rombert (1993), Carvalho Varela $(1975,2005)$, Eiras et al. (1987), Eiras (1990a, 1998), Menezes (1994, 2000), Nunes et al. (2003) and Ramos (2011).

The paper by Eiras (1998) provided a list of all the parasites identified in Portugal's fish until the date of publication. However, given the huge increase in these studies since then, the aforementioned list is now outdated. Therefore, the purpose of this paper is to provide an updated review of fish parasites in Portugal.

\section{Materials and Methods}

The data described in this paper were garnered from a thorough review of the literature. According to the rules of this journal, data contained in Abstracts, Dissertations and Thesis presented at scientific Meetings are not included. However, it should be pointed out that practically all the data contained in Dissertations and Thesis have been published in scientific journals and have therefore been included in this paper, as have numerous Abstracts presented at international scientific Meetings. The name of the host(s) of each parasite species or genera, the parasite's location in the host, site of capture of the host, whenever possible, and references ranked by year of publication are listed. The names of the parasites were checked in the Worms World Register of Marine Species (2016), and are presented in alphabetical order within their respective groups (Isopod crustaceans are placed at the end of the list). The names of fish species were updated, according to FishBase, by Froese \& Pauley (2016). Whenever the location of the parasite in the host is not indicated, this means it was not described by the authors. The capture sites of marine fish are not included because, in most cases, they are unknown - fish are usually captured along the coast, sometimes very far from the port where they are landed, making it impossible to determine their origin. To best present the data, the information is divided into several sections: parasites of marine fish and freshwater fish from mainland Portugal, parasites of fish from the Archipelago of Madeira, from the Archipelago of the Azores, and parasites of farmed fish. A general discussion is presented at the end of this paper.

\section{Parasites of marine fish from mainland Portugal}

Flagellata

Amyloodinium sp., Dicentrarchus labrax, gills, Duarte et al. (2000), Menezes (2000).

Ichthyodinium chabelardi Hollande \& J. Cachon, 1952, Sardina pilchardus, eggs, Meneses \& Ré (1991), Silva \& Miranda (1992), Borges et al. (1996), Stratoudakis et al. (2000).

Icthyobodo necator (Henneguy, 1833) Pinto (1928), Dicentrarchus labrax, gills, Santos (1996), Duarte et al. (2000).

\section{Apicomplexa}

Eimeria sardinae (Thélohan, 1890) Reichenow, 1921, Sardina pilchardus, testes, Pinto (1956), Pinto et al. (1961).

Eimeria sp., Dicentrarchus labrax, gut, Santos (1996).

Goussia cruciata (Thélohan, 1892) Labbé, 1896, Trachurus trachurus, liver, Gestal \& Azevedo (2005), MacKenzie et al. (2008).

G. lusca Gestal \& Azevedo (2006), Trisopterus luscus, liver, Gestal \& Azevedo (2006).

Haemogregarina bigemina Laveran \& Mesnil, 1901, Lipophrys pholis, Coryphoblennius galerita, blood, Sarasquete \& Eiras (1985), Eiras (1987a, b), Eiras \& Davies (1991), Davies et al. (1994), Dias et al. (2005).

\section{Ciliophora}

Cryptocaryon irritans Brown, 1951, Halobatrachus didactylus (=Batrachus didactylus), Diplodus sargus, Caranx ascensiones, Dicentrarchus labrax, Trigla sp., gills, tegument, Grazina Freitas et al. (1986). 
Tetrahymena sp., Dicentrarchus labrax, Solea vulgaris, tegument, Grazina Freitas et al. (1986).

Trichodina sp., Dicentrarchus labrax, Diplodus sargus, gills, Grazina Freitas et al. (1986), Santos (1996), Duarte et al. (2000).

\section{Myxozoa}

Ceratomyxa diplodae Lubat, Radujkovic, Marques \& Bouix (1989), Dicentrarchus labrax, gall bladder, Santos (1996).

C. labracis Sitjà-Bobadilla \& Alvarez-Pellitero (1993), Dicentrarchus labrax, gall bladder, Santos (1996).

Ceratomyxa sp., Aphanopus carbo, Pagellus bogaraveo, gall

bladder, Santos et al. (2009), Hermida et al. (2013b).

Myxobilatus sp., Dicentrarchus labrax, lumen of kidney ducts and urinary bladder, Santos (1996).

Myxobolus sp., Mugil cephalus, tegument, Menezes (1984).

Kudoa sp., Sardina pilchardus, Merluccius merluccius, Trachurus trachurus, muscles, Cruz e Silva \& Grazina Freitas (1984), Grazina Freitas et al. (1986), Menezes et al. (1990b), Gilman \& Eiras (1998), Cruz et al. (2003).

Zschokkela mugilis Sitjà-Bobadilla \& Alvarez-Pellitero (1993), Nereis diversicolor, coelom, Rangel et al. (2009).

\section{Monogenea}

Amphibdella torpedinis Chatin, 1874, Torpedo marmorata, gills, Kearn \& Vasconcelos (1979).

Ancyrocephalus sp., Mugilsp., gills, Kearn \& Vasconcelos (1979). Cemocotyle trachuri Dillon \& Hargis (1965), Trachurus trachurus, gills, MacKenzie et al. (2008).

Choricotyle chrysophryi Van Beneden \& Hesse, 1863, Pagellus bogaraveo, gills, Hermida et al. (2013a, b).

Diclidophora luscae (Van Beneden \& Hesse, 1864) Diesing, 1858, gills, Trisopterus luscus, Kearn \& Vasconcelos (1979).

Diplectanum aequans (Wagner, 1857) Diesing, 1858, Dicentrarchus labrax, gills, Kearn \& Vasconcelos (1979). Santos (1996), Duarte et al. (2000).

Empruthotrema raiae (MacCallum, 1916) Johnston \& Tiegs, 1922, Raja microocellata, gills, Kearn \& Vasconcelos (1979).

E. torpedinis Kearn (1976), Torpedo marmorata, gills, Kearn \& Vasconcelos (1979).

Entobdella soleae (Van Beneden \& Hesse, 1864) Johnston, 1929, Dicologlossa cuneata, Solea senegalensis, S. solea, skin, Carvalho Varela \& Cunha-Ferreira (1987), Kearn \& Vasconcelos (1979), Marques et al. (2006c, 2009, 2011).

Epicotyle torpedinis Price, 1942, Torpedo marmorata, gills, Kearn \& Vasconcelos (1979).

Gastrocotyle trachuri Van Beneden \& Hesse, 1863, Trachurus picturatus, T. trachurus, stomach, MacKenzie et al. (2008), Hermida et al. (2015).

Grubea cochlear Diesing, 1858, Scomber scombrus, gills, Rego et al. (1985), Castro \& Santos (2013).

Gyrodactylus elegans Von Nordman, 1832, Gaidropsarus sp., Gobius niger, Solea solea, Trisopterus luscus, gills, Carvalho Varela et al. (1981), Kearn \& Vasconcelos (1979).
Gyrodactylus sp., Pegusa lascaris (=Solea lascaris), Solea solea, gills, Carvalho Varela \& Cunha-Ferreira (1987), Marques et al. (2006b, c, 2009, 2011).

Haliotrema balisticus (Hargis, 1955) Looss, 1902, Mugil cephalus, gills, Kearn \& Vasconcelos (1979).

Heteraxinoides atlanticus Gaevskaya \& Kovaliova (1979), Trachurus trachurus, gills, MacKenzie et al. (2008).

Heterocotyle pastinacae Scott, 1904, Dasyatis pastinaca, gills, Kearn \& Vasconcelos (1979).

Hexabothrium monteiroi Tendeiro (1955), Squalus blainville, gills, Tendeiro \& Valdez (1955b).

Kuhnia scombri (Kuhn, 1829) Sproston (1945), Scomber scombrus, gills, Kearn \& Vasconcelos (1979), Rego et al. (1985), Castro \& Santos (2013).

K. sprostonae Price (1961), Scomber scombrus, pseudobranchs, Castro \& Santos (2013).

Lamellodiscus virgula Euzet \& Oliver (1967), Pagellus bogaraveo, gills, Hermida et al. (2013a, b).

Lamellodiscus sp., Coris julis, Spondyliosoma cantharus, gills, Kearn \& Vasconcelos (1979).

Leptocotyle minor (Monticelli, 1888) Gallen (1937), Scyliorhinus sp., tegument, Kearn \& Vasconcelos (1979).

Mazocraes vilelai Tendeiro \& Valdez (1955a), Alosa alosa, gills, Tendeiro \& Valdez (1955b).

Microcotyle sp., Coris julis, Diplodus sp., Pomadasys incisus (=Pomadasys bennedetti), Pomatomus saltator, Sparus aurata, Spondyliosoma cantharus, gills, Kearn \& Vasconcelos (1979).

Erpocotyle catenulata (Guberlet, 1933), Mustelus mustelus, gills, Tendeiro \& Valdez (1955b).

Neonchocotyle pastinacae Ktari \& Maillard (1972), Dasyatis pastinaca, gills, Kearn \& Vasconcelos (1979).

Pseudaxine trachuri Parona \& Perugia, 1889, Trachurus picturatus, T. trachurus, gills, MacKenzie et al. (2008), Hermida et al. (2015).

Octoplectanocotyla aphanopi Pascoe (1987), Aphanopus carbo, gills, Santos et al. (2009).

Serranicotyle labracis (Van Beneden \& Hesse, 1863) Maillard, Euzet \& Silan, 1988, Dicentrarchus labrax, gills, Santos (1996).

Thaumatocotyle concinna Scott, 1904, Dasyatis pastinaca, nasal fossae, Kearn \& Vasconcelos (1979).

Trochopus pini (Van Beneden \& Hesse, 1863) Massa, 1903, Lepidotrigla cavillone, gills, Buhrnheim et al. (1973), Kearn \& Vasconcelos (1979).

Winkenthughesia bramae (Parona \& Perugia, 1896) Bychovsky, 1957, Brama brama, gills, Kearn \& Vasconcelos (1979).

\section{Digenea}

Acanthostomum sp., Pomatoschistus microps, digestive tract, Costa JL et al. (2012).

Brachyenteron helicoleni Bray \& Kuchta, 2006, Pagellus bogaraveo, intestine, Hermida et al. (2013b, 2014).

Bucephalus baeri Maillard \& Saad-Fares, 1981, Dicentrarchus labrax, intestine, Santos (1996).

B. minimus (Stossich, 1887), Mugil cephalus, heart, liver, spleen, Pina et al. (2009). 
Cryptocotyle lingua (Creplin, 1825), Pleuronectes platessa, digestive tract, CarvalhoVarela et al. (1981).

Cryptocotyle sp., Pomatoschistus microps, fins (Freitas et al. 2009), Costa JL et al. (2012).

Derogenes varicus (Müller, 1784) Looss, 1901, Dicentrarchus labrax, Dicologlossa cuneata, Microchirus azevia, M. variegatus, Pagellus bogaraveo, Platichthys flesus, Scophthalmus rhombus, Solea kleinii, Pegusa lascaris (=Solea lascaris), S. senegalensis, digestive tract, gills, Carvalho Varela \& Cunha-Ferreira (1987), Santos (1996), Marques et al. (2006a, c, 2009, 2010, 2011), Hermida et al. (2013b, 2014).

Diplectanum aequans (Wagener, 1857) Diesing, 1958, Dicentrarchus labrax, gills, Grazina Freitas et al. (1986).

Didymobothrium rudolphi (Monticelli, 1890), Pegusa lascaris (=Solea lascaris), Marques \& Cabral (2007).

Diphterostomum vividum (Nicoll, 1912) Bray \& Gibson, 1986, Pagellus bogaraveo, intestine, Hermida et al. (2013b, 2014).

Diplostomum sp., Platichthys flesus, eyes, Cavaleiro et al. (2012).

Dolichoenterum manteri Tendeiro, 1955, Conger conger, intestine,

Tendeiro (1955). Ectenurus lepidus Looss, 1907, Solea solea, Trachurus picturatus, T. trachurus, stomach, Carvalho Varela \& Cunha-Ferreira (1987), MacKenzie et al. (2008), Hermida et al. (2015).

Glomericirrus macrouri (Gaevskaya, 1973) Gaevskaya, 1979, Pagellus bogaraveo, Hermida et al. (2013b).

Haploporus benedeni (Stossich, 1887), Mugil cephalus, digestive tract, Carvalho Varela et al. (1981).

Helicometra fasciata (Rudolphi, 1819) Odhner, 1902, Lepidorhombus boscii, Lipophrys pholis, digestive tract, intestine, Santos \& Eiras (1995), Marques et al. (2006c, 2009, 2010, 2011).

Hemiptera sp., Microchirus azevia, M. variegatus, Pegusa lascaris (=Solea lascaris), S. senegalensis, digestive tract, Marques et al. (2006b, c, 2009, 2011).

Hemiurus appendiculatus (Rudolphi, 1802) Looss, 1899, Alosa alosa, A. fallax, Pomatoschistus microps, stomach, Tendeiro \& Valdez (1955a), Rodrigues et al. (1972), Costa JL et al. (2012).

H. communis Odhner, 1905, Dicentrarchus Labrax, Pagellus bogaraveo, Solea solea, stomach, Carvalho Varela \& Cunha-Ferreira (1987), Santos (1996), Hermida et al. (2013b, 2014).

Homalometron galaicus SanMartin, Alvarez, Quintero \& Paniagua, 1995, Dicologlossa cuneata, Microchirus azevia, M. variegatus, Solea senegalensis, digestive tract, Marques et al. (2006b, c, 2009, 2011).

Hypoepaticola sp., Helicolenus dactylopterus, digestive tract, Sequeira et al. (2010).

Lecithochirium furcolabiatum (Jones, 1933) Dawes, 1947, Lipophrys pholis, Pomatoschistus microps, mesenteries, Santos \& Eiras (1995).

L. musculus (Looss, 1907) Nasir \& Diaz, 1971, Dicentrarchus labrax, Pomatoschistus microps, intestine, Santos (1996), Freitas et al. (2009), Costa JL et al. (2012).

L. rufoviridae (Rudolphi, 1819), Arnoglossus laterna, Citharus linguatula, Conger conger, Dicologlossa cuneata, Lepidorhombus boscii, Microchirus azevia, Platichthys flesus, Scophthalmus maximus, S. rhombus, digestive tract, gills, Tendeiro \& Valdez (1955b), Marques et al. (2006b, c, 2009, 2010, 2011).
Lecithochirium sp., Pomatoschistus microps, digestive tract, Freitas et al. (2009).

Lecithocladium excisum (Rudolphi, 1819) Lühe, 1901, Pagellus bogaraveo, Scomber scombrus, gills, stomach, Rego et al. (1985), Hermida et al. (2013b, 2014).

Lecithocladium sp., Helicolenus dactylopterus, digestive tract, Sequeira et al. (2010).

Lepocreadium album (Stossich, 1890), Pagellus bogaraveo, stomach, pyloric cecae, anterior intestine, Hermida et al. (2013b, 2014).

Lomasoma stephanskii Dolffus, 1960, Microchirus variegatus, digestive tract, Marques et al. (2006b, 2009, 2011).

Macvicaria soleae (Dujardin, 1845) Gibson \& Bray, 1982, Dicologlossa cuneata, Microchirus azevia, M. variegatus, Platichthysflesus, Pegusa lascaris (=Solea lascaris), S. senegalensis, S. solea, digestive tract, Durieux et al. (2007), Marques et al. (2006a, b, 2009, 2010, 2011).

Monascus filiformis (Rudolphi, 1819), Trachurus picturatus, intestine, Hermida et al. (2015).

Opechona bacillaris (Molin, 1859) Dollfus, 1927, Scomberscombrus, stomach, intestine, Rodrigues et al. (1972), Rego et al. (1985).

Otodistomum veliporum (Creplin, 1837) Stafford, 1894, Torpedo torpedo, stomach, Tendeiro \& Valdez (1955b).

Otodistomum sp., Synapturichthys kleinii (=Solea kleinii), digestive tract, visceral cavity, Marques et al. (2009, 2011).

Pycnadenoides senegalensis Fischthal \& Thomas, 1972, Pagellus bogaraveo, pyloric cecae, intestine, Hermida et al. (2013b, 2014).

Proctoeces maculatus (Looss, 1901), Platichthys flesus, digestive tract, Marques et al. (2009, 2010, 2011).

Prosorhynchus aculeatus Odhner, 1905, Conger conger, Solea solea, stomach, rectum, branchial arches, muscles, Santos \& Gibson (2002), Durieux et al. (2007), Marques et al. (2006c, 2009), Francisco et al. (2010).

P. crucibulum (Rudolphi, 1819) Odhner, 1905, Conger conger, Dicentrarchus labrax, Dicologlossa cuneata, Synapturichthys kleinit (=Solea kleinii), Pegusa lascaris (=Solea lascaris), S. senegalensis, S. solea, kidney, stomach, muscles, digestive tract, branchial arches, Santos (1996), Santos \& Gibson (2002), Marques et al. (2006b, c, 2009), Durieux et al. (2007), Francisco et al. (2010, 2012).

Prosorhynchus sp., Pomatoschistus microps, muscle, Freitas et al. (2009), Costa JL et al. (2012).

Stephanostomum pristis (Deslongchamps, 1824) Looss, 1899, Trisopterus luscus, intestine, Rodrigues et al. (1975a).

Sterrhurus fusiformis [(=Lecithochirium grandiporum (Rudolphi, 1819) Lühe, 1901], Conger conger, stomach, Tendeiro \& Valdez (1955b).

Tergestia sp., Trachurus picturatus, intestine, Hermida et al. (2015)

Timoniella imbutiforme (Molin, 1859) Brooks, 1980, Dicentrarchus labrax, Solea solea, muscles, Durieux et al. (2007).

T. praeterita (Looss, 1901) Maillard, 1974, Dicentrarchus Labrax, Solea solea, muscles, intestine, Santos (1996), Durieux et al. (2007). Zoogonoides viviparus (Olsson, 1868) Odhner, 1902, Pomatoschistus microps, digestive tract, Costa JL et al. (2012).

Zoogonus rubellus (Olsson, 1868) Odhner, 1902, Dicologlossa cuneata, Platichthys flesus, Solea senegalensis, digestive tract, Marques et al. (2006b, c, 2009, 2010, 2011). 


\section{Cestoda}

Bothriocephalus andresi Porta, 1911, Citharus linguatula, digestive tract, Marques et al. (2009, 2010, 2011).

B. barbatus Renaud, Gabrion \& Pasteur, 1983, Scophthalmus rhombus, digestive tract, Marques et al. (2009, 2010, 2011).

B. clavibothrium Ariola, 1899, Arnoglossus laterna, digestive tract, Marques \& Cabral (2007), Marques et al. (2009, 2010, 2011).

B. gregarious Renaud, Gabrion \& Pasteur, 1983, Scophthalmus maximus, digestive tract, Marques et al. (2009, 2011).

B. scorpii (Müller, 1776), Lepidorhombus boscii, Dicologlossa cuneata, Platichthys flesus, Pegusa lascaris (=Solea lascaris), S. senegalensis, digestive tract, Marques et al. (2006b, c, 2009, 2010, 2011).

Bothriocephalus sp., Pleuronectes platessa, digestive tract, Carvalho Varela et al. (1981).

Clestobothrium crassiceps (Rudolphi, 1819), Merluccius merluccius, intestine, Tendeiro \& Valdez (1955b).

Didymobothrium rudolphi (Monticelli, 1890) Nybelin, 1922, Pegusa lascaris (=Solea lascaris), S. senegalensis, digestive tract, intestine, Marques et al. (2006b, c, 2007, 2009, 2011), Świderski et al. (2010).

Diphyllobothrium sp., Synaptura lusitanica, digestive tract, Marques et al. (2006b, 2009, 2011).

Echeinobothrium sp., Scomber scombrus, intestine wall, Rego et al. (1985).

Hepatoxylon trichiuri (Holten, 1802), Lepidotus caudatus, intestine, gonads, Machado Cruz (1959b), Sequeira et al. (2010).

Heteronybelinia yamagutii (Dollfus, 1960), Aphanopus carbo, digestive tract, Santos et al. (2009).

Grillotia sp., Microchirus variegatus, digestive tract, Marques et al. (2006b, 2009, 2011).

Lacistorhynchus tenuis (Van Beneden, 1858), Scomber scombrus, pyloric cecae, Rego et al. (1985).

Nybelinia lingualis Cuvier, 1817, Citharus linguatula, Dicologlossa cuneata, Lepidorhombus boscii, Microchirus azevia, M. variegatus, Scophthalmus rhombus, Pegusa lascaris (=Solea lascaris), S. senegalensis, S. solea, mesenteries, Durieux et al. (2007), Marques et al. (2006b, c, 2009, 2010, 2011).

Onchobothrium uncinatum (Rudolphi, 1819), Raja undulata, intestine, Tendeiro \& Valdez (1955b).

Progrillotia dasyatidis Beveridge, Neifar \& Euzet, 2004, Dicologlossa cuneata, Halobatrachus didactylus, Lepidorhombus boscii, Microchirus azevia, M. variegatus, Pegusa lascaris (=Solea lascaris), $S$. senegalensis, intestinal lumen, digestive tract, Marques et al. (2005a,b, 2006b, c, 2009, 2010), Costa JL et al. (2012).

Scolex pleuronectis (Müller, 1758), Dicologlossa cuneata, Microchirus azevia, Scomber scombrus, Solea senegalensis, S. solea, Trachurus trachurus, digestive tract, intestine, Rego et al. (1985), Durieux et al. (2007), MacKenzie et al. (2008), Marques et al. (2006b, c, 2009, 2010, 2011).

Sphyriocephalus tergestinus Pintner, 1913, Aphanopus carbo, digestive tract, Santos et al. (2009).

\section{Acanthocephala}

Acanthocephaloides incrassatus (Molin, 1858), Pegusa lascaris (=Solea lascaris), digestive tract, Marques et al. (2006b).
A. geneticus (Buron, Renaud \& Euzet, 1985), Lepidorhombus boscii, Pegusa lascaris (=Solea lascaris), S. senegalensis, digestive tract, Marques et al. (2006b, c, 2009, 2010, 2011).

A. propinquus (Dujardin, 1845), Dicologlossa cuneata, Lepidorhombus boscii, Microchirus azevia, M. variegatus, Monochirus hispidus, Scophthalmus rhombus, Pegusa lascaris (=Solea lascaris), S. senegalensis, S. solea, digestive tract, Durieux et al. (2007), Marques et al. (2006b, c, 2009, 2010, 2011).

Acanthocephalus incrassatus (=Acanthocephaloides incrassatus Molin, 1858), Pegusa lascaris (=Solea lascaris), digestive tract, Marques et al. (2009, 2011).

Bolbosoma vasculosum (Rudolphi, 1819), Aphanopus carbo, digestive tract, Santos et al. (2009).

Bolbosoma sp., Pagellus bogaraveo, intestine, Hermida et al. (2013b).

Echinorhynchus clavula Dujardin, 1845, Solea senegalensis, S. solea, digestive tract, Carvalho-Varela \& Cunha-Ferreira (1987)

Echinorhynchus gadi Zoega in Müller, 1776), Citharus linguatula, Lepidorhombus boscii, digestive tract, Marques \& Cabral (2007), Marques et al. (2006c, 2009, 2010, 2011).

Neoechinorhynchus sp., Mugil cephalus, intestine, Carvalho Varela et al. (1981).

Pomphorhynchus laevis Müller, 1776, Solea senegalensis, S. solea, digestive tract, Carvalho Varela \& Cunha-Ferreira (1987).

Rhadinorhynchus cadenati (Golvan \& Houin, 1964), Trachurus trachurus, intestine, MacKenzie et al. (2008).

R. johni [=Aspersentis johni (Baylis, 1929)], Merluccius merluccius, intestine, Rodrigues et al. (1973, 1975c).

$R$. pristis (Rudolphi, 1802), Pagellus bogaraveo, pyloric cecae, intestine, Hermida et al. (2013b, 2014).

R. tenuicornis [= Telosentis tenuicornis (Linton, 1901)], Scomber scombrus, intestine, Rodrigues et al. (1975b,c), Rego et al. (1985).

Radinorhynchus sp., Citharus linguatula, Microchirus azevia, Pegusa lascaris (=Solea lascaris), Trachurus picturatus, digestive tract, intestine, Marques et al. (2009, 2011), Hermida et al. (2015).

\section{Nematoda}

Anisakis pegreffii Campana-Rouget \& Biocca, 1955, Arnoglossus imperialis, Citharus linguatula, Dicologlossa cuneata, Lepidorhombus boscii, Pagellus bogaraveo, Trachurus trachurus, digestive tract, encapsulated on the outside of the viscera and fish body cavity, Marques et al. (2006a,b 2009), Mattiucci et al. (2008), Hermida et al. (2012b).

A. physeteris (Bayliss, 1923), Pagellus bogaraveo, most larvae encapsulated in fish body cavity, mesenteries, on the ouside of the viscera, Hermida et al. (2012b).

A. simplex s.s., Arnoglossus laterna, A. imperialis, Citharus linguatula, Dicologlossa cuneata, Pagellus bogaraveo, Pegusa lascaris (=Solea lascaris), S. senegalensis, most larvae encapsulated in fish body cavity, mesenteries, on the ouside of the viscera, Marques et al. (2006a, b), Hermida et al. (2012b).

A. simplex (Rudolphi, 1809), Arnoglossus imperialis, A. laterna, Citharus linguatula, Dicologlossa cuneata, Lepidorhombus boscii, L. whiffiagonis, Zeugopterus regius (=Phrynorhombus regius), Scomber scombrus, Synapturichthys kleinii (=Solea kleinii), Pegusa 
lascaris (=Solea lascaris), Trachurus trachurus, digestive tract, body cavity, liver, stomach, Rego et al. (1985), Mattiucci et al. (2008), Marques et al. (2009, 2011).

A. simplex $x$ A. pegreffi, Pagellus bogaraveo, most larvae encapsulated in fish body cavity, mesenteries, on the ouside of the viscera, Hermida et al. (2012b).

A. typica (Diesing, 1860), Pagellus bogaraveo, Platichthys flesus, most larvae encapsulated in fish body cavity, mesenteries, on the ouside of the viscera, digestive tract, Marques et al. (2006a, c, 2009, 2010, 2011), Hermida et al. (2012b).

A. ziphidarum Pagi, Nascetti, Webb, Mattiuci, Cianchi \& Bullini, 1988, Pagellus bogaraveo, most larvae encapsulated in fish body cavity, mesenteries, on the ouside of the viscera, Hermida et al. (2012b).

Anisakis sp., Aphanopus carbo, Conger conger, Helicolenus dactylopterus, Merluccius merluccius, Micromesistius poutassou, Pomatoschistus microps, Sardina pilchardus, Scomber scombrus, Solea sp., Spondyliosoma cantharus, Trachurus picturatus, T. trachurus, Trigla lucerna, Trisopterus luscus, surface of internal organs, visceral cavity, mesentery, liver, muscles, Grazina Freitas et al. (1986), Saraiva et al. (2000b), Silva \& Eiras (2003), MacKenzie et al. (2008), Cruz et al. (2005, 2007, 2009), (Freitas et al. 2009), Santos et al. (2009), Sequeira et al. (2010), Hermida et al. (2015).

Camallanus sp., Pagellus bogaraveo, pyloric cecae, Hermida et al. (2013b, 2014).

Capillaria sp., Citharus linguatula, Dicologlossa cuneata, Platichthys flesus, Synaptura lusitanica, gut lumen, Marques et al. (2011).

Contracaecum aduncum (Rudolphi, 1802), Alosa alosa, Pleuronectes platessa, stomach, intestine, Tendeiro \& Valdez (1955b), Carvalho Varela et al. (1981).

C. gadi (Müller, 1776), Merluccius merluccius, stomach, Tendeiro \& Valdez (1955b).

C. magnum Smedley 1934, [= Hysterothylacium magnum (Smedley, 1934], Solea solea, intestine, Rodrigues et al. (1975a).

Contracaecum sp., Pomatoschistus microps, digestive tract, (Costa JL et al., 2012).

Cristitectus congeri Petter, 1970, Conger conger, stomach, intestine, Saraiva et al. (2000b).

Cucullanus campanae Lebre \& Petter, 1984, Dicologlossa cuneata, Microchirus azevia, M. variegatus, Platichthys flesus, Pegusa lascaris (=Solea lascaris), S. senegalensis, S. solea, digestive tract, Durieux et al. (2007), Marques et al. (2006b, c, 2009, 2010, 2011).

C. heterochrous Rudolphi, 1802, Microchirus azevia, digestive tract, Marques et al. (2006b, c, 2009).

C. hians Dujardin, 1845, Conger conger, intestine, stomach, Tendeiro \& Valdez (1955b), Saraiva et al. (2000b).

C. longispiculum Rodrigues, Carvalho Varela, Rodriges \& Cristófaro, 1973, Conger conger, stomach, intestine, Rodrigues et al. (1973), Saraiva et al. (2000b).

Cucullanus sp., Arnoglossus laterna, gut lumen, Marques et al. (2011).

Dichelyne minutus (Rudolphi, 1819), Platichthys flesus, digestive tract, Marques et al. (2006c, 2009, 2010, 2011).

Goezia sp., Scomber scombrus, intestine, Rego et al. (1985).

Huffmanella sp., Trisopterus luscus, intestine submucosa, Esteves et al. (2009).
Hysterothylacium aduncum (Rudolphi, 1802), Halobatrachus dydactilus, Microchirus variegatus, Platichthys flesus, Pegusa lascaris (=Solea lascaris), Solea solea, Trachurus trachurus, digestive tract, intestine, Carvalho Varela \& Cunha Ferreira (1987), MacKenzie et al. (2008), Marques et al. (2005a, b, 2006c, 2009, 2010, 2011), Costa JL et al. (2012).

Hysterothylacium sp., Solea senegalensis, S. solea, intestine, Carvalho Varela \& Cunha-Ferreira, 1987.

H. reliquens (Norris \& Overstreet, 1975), Microchirus azevia, M. boscanion, $M$. variegatus, digestive tract Marques et al. (2006b, c, 2009, 2011).

Hysterothylacium sp., Microchirus azevia, Micromesistius poutassou, Pagellus bogaraveo, Pegusa lascaris (=Solea lascaris), Trachurus trachurus, digestive tract, liver, muscles, encapsulated in body cavity and outside of the viscera, Cruz et al. (2005, 2007), Marques et al. (2006a, 2009, 2011), Hermida et al. (2012a).

Luzonema cruzi Rodrigues, Varela, Rodrigues, Cristófaro, 1973, Letrhinus atlanticus, intestine, Rodrigues et al. (1973).

Philometra fliformis (Stossich, 1896), Pagellus bogaraveo, intestine, Hermida et al. (2013b).

Proleptus obtusus, Dujardin, 1845, Scyliorhinus canicula, stomach, Rodrigues et al. (1973).

P. robustus (Van Beneden, 1871), Mustelus canis, Raja clavata, stomach, spiral valve, Tendeiro \& Valdez (1955b), Rodrigues et al. (1973).

Raphidascaris acus (Bloch, 1779), Mugil cephalus, Pomatoschistus microps, digestive tract, Carvalho Varela et al. (1981), Costa JL et al. (2012).

Thynnascaris aduncum [(=Hysterothylacium aduncum (Rudolphi, 1802)], Scomber scombrus, stomach, pyloric cecae, testes, intestine, Rego et al. (1985).

\section{Hirudinea}

Caliobdella sp., Solea senegalensis, skin, Marques et al. (2006b, 2009, 2011).

Hemibdella soleae (Van Beneden \& Hesse, 1863), Dicologlossa cuneata, Microchirus azevia, Pegusa lascaris (=Solea lascaris), Synapturichthys kleinii (=Solea kleinii), S. senegalensis, Synaptura lusitanica, skin, Marques et al. (2006b, c, 2009, 2011).

\section{Crustacea}

Acanthochondria cornuta (Müller O.F., 1776), Platichthys flesus, gill chamber, gill arches, Marques \& Cabral (2007), Marques et al. (2006c, 2009, 2011), Cavaleiro \& Santos (2009, 2011).

A. solea (Krøyer, 1838), Pegusa lascaris (=Solea lascaris), S. senegalensis, S. solea, gill arches, Marques et al. (2006b, c, 2009).

Bomolochus soleae Claus, 1864, Dicologlossa cuneata, Synapturichthys kleinii (=Solea kleinii), S. senegalensis, S. solea, gills, Durieux et al. (2007), Marques et al. (2006b, c, 2009, 2011).

Caligus brevicaudatus A. Scott, 1901, Pegusa lascaris (=Solea lascaris), S. senegalensis, S. solea, skin, Marques et al. (2006c, 2009, 2011).

C. dicentrarchi Cabral \& Raibaut 1983, Dicentrarchus labrax, skin, Santos (1996). 
C. elongatus Von Nordman, 1832, Microchirus azevia, M. variegatus, Solea solea, skin, Marques et al. (2006b, c, 2009, 2011).

C. minimus Otto, 1821, Dicentrarchus labrax, mouth cavity and gills, Santos (1996).

C. musaicus Cavaleiro, Santos \& Ho, 2010, Platichthys flesus, skin and fins, Cavaleiro et al. (2010).

C. pageti Russell, 1925, Dicentrarchus labrax, mouth, Santos (1996).

C. pelamydis Krøyer, 1863, Scomber scombrus, Trachurus trachurus, gills, opercula, branchial chamber internal wall, MacKenzie et al. (2008), Castro \& Santos (2013).

Caligus sp., Dicentrarchus labrax, Platichthys flesus, Trachurus picturatus, skin, gills, operculum, Santos (1996), Cavaleiro \& Santos (2009).

Clavellisa scombri (Kurz, 1877), Scomber scombrus, gills, Castro \& Santos (2013).

Colobomatus labracis Delamare Debouteville \& Nunes Ruivo, 1952, Dicentrarchus labrax, pre-opercular channels, Santos (1996).

Ergasilus sp., Dicologlossa cuneata, gills, Marques et al. (2006b, 2009, 2011).

Holobomolochus confusus (Stock, 1953) [= Cresseyus confusus (Stock, 1953)], Platichthys flesus, nasal cavity, Cavaleiro \& Santos (2009).

Lepeophtheirus europaensis Zeddam, Berrebi, Renaud, Raibaut \& Gabrion, 1988, Scophthalmus rhombus, Dicologlossa cuneata, skin, Marques et al. (2006b, 2009, 2011).

L. pectoralis (Müller O.F., 1776), Platichthys flesus, Solea senegalensis, skin, Marques et al. (2006b, c, 2009, 2011), Cavaleiro \& Santos (2007).

Lernanthropus kroyeri Van Beneden, 1851, Dicentrarchus labrax, gills, Santos (1996).

Lernaeocera caparti Machado Cruz, 1959, Merluccius merluccius, gills, Machado Cruz (1959a).

L. lusci (Basset-Smith, 1896), Lepidorhombus boscii, Trisopterus luscus, tegument, Eiras (1984, 1986), Eiras \& Santos (1990, 1992).

Lernaeocera sp., Microchirus azevia, gills, Marques et al. (2006b, c, 2009, 2011).

Peniculus fistula Nordmann, 1832, Pagellus bogaraveo, Trachurus picturatus, T. trachurus, fins, Candeias (1955), MacKenzie et al. (2008), Hermida et al. (2013a, b).

Rebelula edwardsii (Kölliker, 1853) (=Lophoura edwardsii Kölliker, 1853), Coelorhynchus caelorhynchus, behind the posterior part of dorsal fin, Candeias (1952a).

Peroderma cylindricum Heller, 1865, Sardina pilchardus, tegument, Candeias (1952b).

Aega sp., Citharus linguatula, skin, Marques et al. (2009).

Gnathia maxillaris (Montagu, 1804), Coryphoblennius galerita, Lipophrys pholis, skin, Davies et al. (1994).

Gnathia sp., Helicolenus dactylopterus, Lepidorhombus boscii, Microchirus azevia, Pagellus bogaraveo, Platichthys flesus, Pegusa lascaris (=Solea lascaris), S. solea, Zeugopterus punctatus, mouth, oesophagous, gills, gill chamber, tegument, fins, Sequeira et al. (2010), Marques et al. (2006b, c, 2009, 2011), Hermida et al. (2013a, b).

Nerocila orbignyi (Guérin-Méneville, 1832), Halobatrachus dydactylus, Monochirus hispidus, Platichthys flesus, skin, Marques et al. (2005a, 2009), Cavaleiro \& Santos (2009).
Paragnathia formica (Hesse, 1864), Mugil cephalus, tegument, Menezes \& Cascalho (1984).

Rocinela danmoniensis Leach, 1818, Pagellus bogaraveo, tegument, Hermida et al. (2013a, b).

Rocinela sp., Arnoglossus laterna, Citharus linguatula, Microchirus azevia, Scophthalmus maximus, S. rhombus, Pegusa lascaris (=Solea lascaris), S. senegalensis, Synaptura lusitanica, skin, Marques et al. (2006b, c, 2009, 2011).

\section{Parasites of freshwater fish from mainland Portugal}

Flagellata

Trypanosoma granulosum Laveran \& Mesnil, 1902, Anguilla anguilla, blood, rivers Ancora, Ardena, Este, Febros, Olivais and Tamente, França (1907), Eiras (1988, 1990b), Saraiva \& Eiras (1996), Cruz \& Eiras (1997), Saraiva \& Chubb (1989).

\section{Apicomplexa}

Babesiosoma bettencourti (França, 1908), Anguilla anguilla, blood, rivers Ancora, Este, Febros and Olivais, Cruz \& Davies (1998).

\section{Ciliophora}

Ichthyophthirius multifiliis Fouquet, 1876, Anguilla anguilla, skin and gills, river Este, Saraiva \& Eiras (1996), Saraiva \& Chubb (1989).

Trichodina jadranica Raabe, 1958, Anguilla anguilla, gills, river Este, Saraiva \& Eiras (1996), Saraiva \& Chubb (1989).

\section{Myxozoa}

Actinomyxon Actinospore type, Tubifex tubifex, host fish unknown, river Sousa, Székely et al. (2005).

Hoferellus gilsoni (Debaisieux, 1925), Anguilla anguilla, urinary bladder, river Este, Saraiva \& Eiras (1996), Saraiva (1995), Saraiva et al. (1998).

Myxidium giardi Cépède, 1906, Anguilla anguilla, gills and other organs, Aveiro lagoon, river Este, Azevedo et al. (1989), Saraiva (1995), Saraiva \& Eiras (1996), Saraiva \& Chubb (1989), Hermida et al. (2008).

M. rhodei Léger, 1905, Pseudochondrostoma polylepis (=Chondrostoma polylepis), kidney, urinary ducts, river Ave, Saraiva et al. (2000a).

Myxobolus branchialis (Markewitsch, 1932), Luciobarbus bocagei, gills, river Sousa, Molnár et al. (2012).

M. branchilateralis Molnár, Eszterbauer, Marton, Székely \& Eiras, 2012, Luciobarbus bocagei, gills, river Sousa, Molnár et al. (2012).

M. cutanei Alvarez-Pellitero \& González-Lanza, 1985, Luciobarbus bocagei, fins, scales, river Sousa, Molnár et al. (2012).

M. cyprini Doflein, 1898, Pseudochondrostoma polylepis (=Chondrostoma polylepis), muscle, kidney, gills, urinary bladder, Ermal reservoir, Cruz et al. (1998). 
M. musculi Keysselit, 1908, Luciobarbus bocagei, muscles, river Sousa, Molnár et al. (2012).

M. pfeifferi (Thélohan, 1895), Luciobarbus bocagei, muscles, brânquias, river Sousa, Molnár et al. (2012).

M. portucalensis Saraiva \& Molnár, 1990, Anguilla anguilla, edge of fins, river Este, Saraiva \& Molnár (1990), Saraiva (1995), Saraiva et al. (1998), Saraiva \& Eiras (1996).

M. tauricus Miroshnichenko, 1979, Luciobarbus bocagei, fins, muscles, river Sousa, Molnár et al. (2012).

Myxobolus sp., Anguilla anguilla, Pseudochondrostoma polylepis (=Chondrostoma polylepis), Squalius cephalus (=Leuciscus cephalus), muscles, gills, kidney, fins, urinary ducts, liver, river Este, Saraiva \& Chubb (1989), Cruz et al. (2000).

Zschokkela stettinensis Wierbicka, 1987, Anguilla anguilla, urinary bladder, river Este, Saraiva (1995), Saraiva et al. (1998), Saraiva \& Eiras (1996).

\section{Monogenea}

Pseudodactylogyrus anguillae (Yin \& Sproston, 1948), Anguilla anguilla, gills, rivers Este and Sousa, Aveiro lagoon, Saraiva (1995), Saraiva et al. (1998), Rodrigues \& Saraiva (1996), Saraiva \& Chubb (1989), Hermida et al. (2008).

Pseudodactylogyrus bini (Kikuchi, 1929), Anguilla anguilla, rivers Este and Sousa, gills, Saraiva (1995), Rodrigues \& Saraiva (1996), Saraiva et al. (1998).

\section{Digenea}

Deropristis inflata (Molin, 1859), Anguilla anguilla, stomach, intestine, Aveiro lagoon, Hermida et al. (2008).

Helicometra fasciata (Rudolphi, 1819) Odhner, 1902, Anguilla anguilla, intestine, Aveiro lagoon, Hermida et al. (2008).

Lecithochirium rufoviridae (Rudolphi, 1819) Lühe, 1902, Anguilla anguilla, stomach, Aveiro lagoon, Hermida et al. (2008).

Lecithochirium sp., Anguilla anguilla, gills, Aveiro lagoon, Hermida et al. (2008).

Podocotyle sp., Anguilla anguilla, intestine, Aveiro lagoon, Hermida et al. (2008).

\section{Cestoda}

Bothriocephalus claviceps (Goeze, 1782), Anguilla anguilla, intestine, river Este, Saraiva \& Eiras (1996), Saraiva \& Chubb (1989), Saraiva et al. (2005).

Khawia baltica Szidat, 1941, Luciobarbus bocagei (=Barbus barbus bocagei), intestine, rivers Este, Lima, Paiva and Sousa, Chubb et al. (1997).

\section{Acanthocephala}

Acanthocephalus clavula Dujardin, 1845, Anguilla anguilla, intestine, river Este, Aveiro lagoon, Saraiva \& Eiras (1996), Saraiva et al. (2005), Hermida et al. (2008).
A. lucii (Müller, 1777), Anguilla anguilla, intestine, river Este, Saraiva \& Chubb (1989).

Pomphorhynchus laevis (Müller, 1776), Anguilla anguilla, intestine, Aveiro lagoon, Hermida et al. (2008).

\section{Nematoda}

Anguillicola crassus Kuwahara, Niimi \& Hagaki, 1974, Anguilla anguilla, swimbladder, Aveiro lagoon, Cruz \& Silva et al. (1992), Cardoso \& Saraiva (1998).

Anisakis pegreffii Campana-Rouget \& Biocca, 1955, Alosa alosa, A. fallax, visceral organs, rivers Minho and Mondego, Bao et al. (2015).

A. simplex (Rudolphi, 1809), Alosa alosa, A. fallax, visceral organs, rivers Minho and Mondego, Bao et al. (2015).

Contracaecum sp., Anguilla anguilla, intestinal wall, Aveiro lagoon, Hermida et al. (2008).

Cucullanus truttae Fabricius, 1794, Anguilla anguilla, intestine, river Este, Saraiva \& Eiras (1996), Saraiva et al. (2005).

Paraquimperia tenerrima (Linstow, 1878), Anguilla anguilla, intestine, river Este, Saraiva \& Chubb (1989), Saraiva et al. (2005).

Philometra ovata (Zeder, 1803), Gobio lozanoi, body cavity, river Febros, Saraiva et al. (2008).

Pseudocapillaria tomentosa (Dujardin, 1843), Anguilla anguilla, intestine, river Este, Saraiva \& Eiras (1996).

Rhabdochona anguillae Spaul, 1927, Anguilla anguilla, intestine, river Sousa, Carvalho Saraiva \& Moravéc (1998), Saraiva et al. (2002c).

R. gnedini Skrjabin, 1946, Luciobarbus bocagei (=Barbus barbus bocagei), intestine, river Sousa, Saraiva et al. (2002).

Raphidascaris acus (Bloch, 1779), Salmo trutta fario, intestine, river Mezio, Eiras \& Reichenbach-Klinke (1982), Saraiva et al. (1998).

Spinitectus inermis (Zeder, 1800), Anguilla anguilla, intestine, river Sousa, Saraiva et al. (2002a, b, 2005).

\section{Crustacea}

Acanthochondria cornuta (Müller, 1776), Platichthys flesus, tegument, fins, gill arches, nasal cavities, pseudobranchiae, Viana do Castelo, Matosinhos, Aveiro, Figueira da Foz, Cavaleiro \& Santos (2007).

Caligus diaphanus Nordmann, 1832, Platichthys flesus, tegument, Aveiro, Cavaleiro \& Santos (2007).

Caligus sp., Platichthys flesus, tegument, fins, Matosinhos, Cavaleiro \& Santos (2007).

Ergasilus gibbus Nordmann, 1832, Anguilla anguilla, gills, river Este, Aveiro lagoon, Saraiva (1995, 1996), Saraiva \& Eiras (1996), Saraiva \& Chubb (1989), Hermida et al. (2008).

Holomobolochus confusus (Hemmingsen \& MacKenzie 2001), Platichthys flesus, nasal cavities, Matosinhos, Cavaleiro \& Santos (2007).

Lepeophtheirus pectoralis (Müller, 1776), Platichthys flesus, tegument, fins, Viana do Castelo, Matosinhos, Aveiro Figueira da Foz, Cavaleiro \& Santos (2007). 
L. salmonis (Krøyer, 1838), Salmo trutta, tegument, river Minho catchment, Bao et al. (2016).

Lernaea cyprinacea Linnaeus 1758, Salmo trutta, tegument, river Minho catchment, Bao et al. (2016).

Lernaea sp., Leuciscus cephalus, skin, river Frio, Saraiva \& Valente (1988).

Nerocila orbignyi (Guérin-Méneville, 1832), Platichthys flesus, fins, gill arches, Matosinhos, Cavaleiro \& Santos (2007).

\section{Parasites of marine fish from the Archipelago of Madeira}

\section{Apicomplexa}

Goussia cruciata (Thélohan, 1894) Labbé, 1896, Trachurus picturatus, liver, Costa et al. (2013b).

\section{Myxozoa}

Ceratomyxa sparusaurati Sitjà-Bobadilla, Palenzuela \& Alvarez-Pellitero, 1995, Sparus aurata, gall bladder, Costa et al. (1998).

C. tenuispora Kabata, 1960, Aphanopus carbo, gall bladder, Costa et al. (1996), Casal et al. (2007).

Ceratomyxa sp., Aphanopus carbo, Pagellus bogaraveo, gall bladder, Santos et al. (2009), Hermida et al. (2013b).

\section{Monogenea}

Choricotyle chrysophryi Van Beneden \& Hesse, 1863, Pagellus bogaraveo, gills, Hermida et al. (2013a, b).

Grubea cochlear Diesing, 1858, Scomber japonicus, gills, Costa et al. (2007), Oliva et al. (2008).

Heteraxinoides atlanticus Gaevskaya \& Kovaliova, 1979, Trachurus picturatus, gills, Costa G et al. (2012).

Kuhnia scombri Kuhn, 1829 (Sproston, 1945), Scomber japonicus, gills, Costa et al. (2007), Oliva et al. (2008).

K. sprostonae Price, 1961, Scomber japonicus, gills, Costa et al. (2007).

K. scombercolias Nasir \& Fuentes Zambrano, 1983, Scomber japonicus, gills, Costa et al. (2007), Oliva et al. (2008).

Lamellodiscus virgula Euzet \& Oliver, 1967, Pagellus bogaraveo, gills, Hermida et al. (2013a, b).

Octoplectanocotyla aphanopi Pascoe, 1987, Aphanopus carbo, gills, Santos et al. (2009).

Pseudaxine trachuri Parona \& Perugia, 1889, Trachurus picturatus, gills, Costa G et al. (2012).

Pseudokuhnia minor (Goto, 1984), Scomber japonicus, gills, Costa et al. (2007), Oliva et al. (2008).

\section{Digenea}

Diphterostomum vividum (Nicoll, 1912) Bray \& Gibson, 1986, Pagellus bogaraveo, Hermida et al. (2013b).
Halvorsenius exilis Gibson, MacKenzie \& Cottle, 1981, Scomber japonicus, intestine, Oliva et al. (2008).

Lecithochirium fusiforme Lühe, 1901, [= Lecithochirium grandiporum (Rudolphi, 1819) Lühe, 1901] Conger conger, stomach, Costa et al. (2009b).

L. musculus (Looss, 1907) Nasir \& Diaz, 1971, Conger conger, stomach, Costa et al. (2009b).

Nematobothrium scombri (Taschenberg, 1879), Scomber japonicus, intestine, Oliva et al. (2008).

Pachycreadium carnosum (Rudolphi, 1819) Cortini \& Ferretti, 1959, Pagellus bogaraveo, pyloric cecae, intestine, Hermida et al. (2013b, 2014).

Prodistomum orientale (Layman, 1930) Bray \& Gibson, 1990, Scomber japonicus, intestine, Oliva et al. (2008).

Schikhobalotrema longivesiculatum Orecchia \& Paggi, 1975, Parablennius parvicornis, intestine, Gibson \& Costa (1997).

\section{Cestoda}

Campbelliella heteropoeciloacantha Palm, 2004, Aphanopus carbo, digestive tract, Santos et al. (2009).

Gryllotia sp., Cataetyx laticeps, in cysts on the outer wall of stomach, Costa et al. (2016).

Heteronybelinia yamagutii Dollfus, 1960, Aphanopus carbo, digestive tract, Santos et al. (2009).

Heteronybelinia sp., Aphanopus carbo, digestive tract, Santos et al. (2009).

Nybelinia lingualis Cuvier, 1817, Aphanopus carbo, Pseudaxine trachuris, Trachurus picturatus, stomach wall, digestive tract, Santos et al. (2009), Costa G et al. (1996, 2003b, 2012, 2013b).

N. thyrsites Korotaeva, 1971, Aphanopus carbo, Trachurus picturatus, external stomach wall, digestive tract, Costa et al. (2003b), Santos et al. (2009).

Nybelinia sp., Mauligobius maderensis, Scomber japonicus, intestine, Gibson \& Costa (1997), Oliva et al. (2008).

Pseudogrillotia epinepheli Scholz, Garippa \& Scalla, 1993, Serranus atricauda, inside nodules attached to the wall of internal organs, Costa et al. (2013a).

Scolex pleuronectis Müller, 1788, Abudefduf luridus, Scomber japonicus, Sphoeroides marmoratus, Thalassoma pavo, intestine, Costa \& Biscoito (2003), Oliva et al. (2008).

Sphyriocephalus tergestinus Pintner, 1913, Aphanopus carbo, stomach wall, digestive tract, Costa et al. (2003b, 1996), Santos et al. (2009).

Tentacularia coryphaenae (Bosc, 1802), Aphanopus carbo, Centrophorus squamosus, Scomber japonicus, Trachurus picturatus, stomach wall, Costa et al. (1996), Oliva et al. (2008), Santos et al. (2009), Costa G et al. (2003b, 2012, 2014).

Acanthocephala

Bolbosoma vasculosum (Rudolphi, 1819), Aphanopus carbo, Scomber japonicus, Serranus atricauda, Trachurus picturatus, connective tissues of viscera, digestive tract, Oliva et al. (2008), Santos et al. (2009), Costa G et al. (2000, 2012, 2013a). 
Bolbosoma sp., Aphanopus carbo, Pagellus bogaraveo, Scomber japonicus, external stomach wall, Costa et al. (1996), Oliva et al. (2008), Hermida et al. (2013b).

Rhadinorhynchus cadenati (Golvan \& Houin, 1964), Trachurus picturatus, intestine, Costa et al. (2013b).

$R$. pristis (Rudolphi, 1802), Conger conger, Scomber japonicus, stomach, Oliva et al. (2008), Costa et al. (2004b, 2009b).

\section{Nematoda}

Anisakis brevispiculata Dollfus, 1968, Aphanopus carbo, most larvae encapsulated in fish body cavity, mesenteries, on the ouside of the viscera, Costa et al. (2003a).

A. pegreffii Campana Rouget \& Biocca, 1955, Aphanopus carbo, Pagellus bogaraveo, Scomber japonicus, Trachurus picturatus, most larvae encapsulated in fish body cavity, mesenteries, on the ouside of the viscera, Costa et al. (2003a, 2004a), Pontes et al. (2005), Hermida et al. (2012b).

A. physeteris (Bayliss, 1923), Pagellus bogaraveo, Scomber japonicus, most larvae encapsulated in fish body cavity, mesenteries, on the ouside of the viscera, Costa et al. (2003a), Pontes et al. (2005), Hermida et al. (2012b).

A. simplex s.l., Pagellus bogaraveo, most larvae encapsulated in fish body cavity, mesenteries, on the ouside of the viscera, Costa et al. (2004a, 2009b), Hermida et al. (2012b).

A. simplex s.s., Aphanopus carbo, Centrophorus squamosus, Conger conger, Pagellus bogaraveo, Scomber japonicus, Trachurus picturatus, most larvae encapsulated in fish body cavity, mesenteries, on the ouside of the viscera, Pontes et al. (2005), Hermida et al. (2012b), Costa et al. (2003a, 2004a, 2014).

A. typica (Diesing, 1860), Pagellus bogaraveo, Scomber japonicus, Trachurus picturatus, most larvae encapsulated in fish body cavity, mesenteries, on the ouside of the viscera, Mattiucci et al. (2002), Costa et al. (2003a), Pontes et al. (2005), Hermida et al. (2012b).

A. ziphidarum Paggi, Nascetti, Webb, Mattiucci, Cianchi \& Bullini, 1988, Aphanopus carbo, Pagellus bogaraveo, Scomber japonicus, Trachurus picturatus, most larvae encapsulated in fish body cavity, mesenteries, on the ouside of the viscera, Costa et al. (2003a), Pontes et al. (2005), Hermida et al. (2012b).

Anisakis sp., Aphanopus carbo, Helicolenus dactylopterus, Pagellus bogaraveo, Scomber japonicus, Trachurus picturatus, most larvae encapsulated in fish body cavity, mesenteries, on the ouside of the viscera, Costa et al. (1996), Pontes et al. (2005), Oliva et al. (2008), Cruz et al. (2009), Santos et al. (2009), Sequeira et al. (2010), Hermida et al. (2012b), Costa G et al. (2003a, 2012, 2013b).

Clavellisa scombri (Kurz, 1877), Scomber japonicus, intestine, Oliva et al. (2008).

Cristitectus congeri Petter, 1970, Conger conger, stomach, Costa et al. (2009b).

Hysterothylacium fabri (Rudolphi, 1819), Scomber japonicus, intestine, Oliva et al. (2008).

Hysterothylacium sp., Boops boops, Diplodus vulgaris, Pagellus bogaraveo, Scomber japonicus, Serranus atricauda, Thalassoma pavo, most larvae encapsulated in fish body cavity, mesenteries, on the ouside of the viscera, Costa \& Biscoito (2003), Oliva et al. (2008), Hermida et al. (2012b), Costa et al. (2004a, 2013a).
Onchophora melanocephala (Rudolphi, 1819), Scomber japonicus, body cavity, Costa et al. (2009a).

Onchophora sp., Scomber japonicus, intestine, Oliva et al. (2008).

Procammallanus (Spirocamallanus) halitrophus Fusco \& Overstreet, 1978, Serranus atricauda, intestine, Costa et al. (2013a).

Pseudoterranova ceticola (Deardorff \& Overstreet, 1981),

Centrophorus squamosus, stomach, Costa et al. (2014).

Spirocamallanus sp., Mauligobius maderensis, intestine, Gibson \& Costa (1997).

\section{Hirudinea}

Hirudinella ventricosa (Pallas, 1774) Baird, 1853, Helicolenus dactylopterus, gonads, Sequeira et al. (2010).

\section{Crustacea}

Hatschekia pagellibogneravei (Hesse, 1878), Pagellus bogaraveo, gills, Hermida et al. (2012a, 2013a, b).

Aega deshaysiana (=Aegapheles deshaysiana) (Milne Edwards, 1840), Pagellus bogaraveo, tegument, Hermida et al. (2013a, b).

Gnathia maxillaris (Montagu, 1808), Coryphoblennius galerita, Lipophrys pholis, skin, Davies et al. (1994).

Gnathia sp., Helicolenus dactylopterus, Pagellus bogaraveo, mouth, oesophagous, gills, gill chamber, tegument, fins, Sequeira et al. (2010), Hermida et al. (2013a, b).

Meinertia oestroides (Risso, 1826), Boops boops, Costa \& Biscoito (2003).

Rocinela danmoniensis Leach, 1818, Pagellus bogaraveo, tegument, Hermida et al. (2013a, b)

\section{Parasites of marine fish from the Archipelago of the Azores}

Myxozoa

Ceratomyxa sp., Aphanopus carbo, gall bladder, Santos et al. (2009).

\section{Monogenea}

Choricotyle chrysophryi Van Beneden \& Hesse, 1863, Pagellus bogaraveo, gills, Hermida et al. (2013a, b).

Lamellodiscus virgula Euzet \& Oliver, 1967, Pagellus bogaraveo, gills, Hermida et al. (2013a, b).

\section{Digenea}

Accacladocoelium petasiporum Odhner, 1928, Pagellus bogaraveo, stomach, Hermida et al. (2013b, 2014).

Derogenes varicus (Müller, 1784) Looss, 1901, Pagellus bogaraveo, stomach, Hermida et al. (2013b, 2014).

Lecithochirium musculus (Looss, 1907) Nasir \& Diaz, 1971, Aphanopus carbo, digestive tract, Santos et al. (2009). 
Lecithocladium excisum (Rudolphi, 1819) Lühe, 1901, Pagellus bogaraveo, stomach, Hermida et al. (2013b, 2014).

Lepocreadium album (Stossich, 1890), Pagellus bogaraveo, stomach, pyloric cecae, anterior intestine, Hermida et al. (2013b, 2014).

Odrhenium sp., Helicolenus dactylopterus, mesenteries, Sequeira et al. (2010).

Pachycreadium carnosum (Rudolphi, 1819) Cortini \& Ferretti, 1859, Pagellus bogaraveo, pyloric cecae, Hermida et al. (2013b, 2014).

Pycnadenoides senegalensis Fischthal \& Thomas, 1972, Pagellus bogaraveo, pyloric cecae, intestine, Hermida et al. (2013b, 2014).

\section{Cestoda}

Heteronybelinia yamagutii, (Dollfus, 1960), Aphanopus carbo, digestive tract, Santos et al. (2009).

Nybelinia lingualis Cuvier, 1817, Aphanopus carbo, digestive tract, Santos et al. (2009).

\section{Acanthocephala}

Bolbosoma vasculosum (Rudolphi, 1819), Aphanopus carbo, digestive tract, Santos et al. (2009).

Bolbosoma sp., Pagellus bogaraveo, intestine, Hermida et al. (2013b).

Rhadinorhynchus pristis (Rudolphi, 1802), Pagellus bogaraveo, stomach, pyloric cecae, intestine, Hermida et al. (2013b, 2014).

\section{Nematoda}

Anisakis pegreffii Campana-Rouget \& Biocca, 1955, Pagellus bogaraveo, most larvae encapsulated in fish body cavity, mesenteries, on the ouside of the viscera, Hermida et al. (2012b).

A. physeteris (Bayliss, 1923), Pagellus bogaraveo, most larvae encapsulated in fish body cavity, mesenteries, on the ouside of the viscera, Hermida et al. (2012b).

A. simplex s.l., Pagellus bogaraveo, most larvae encapsulated in fish body cavity, mesenteries, on the ouside of the viscera, Hermida et al. (2012b).

A. simplex s.s., Pagellus bogaraveo, most larvae encapsulated in fish body cavity, mesenteries, on the ouside of the viscera, Hermida et al. (2012b).

A. simplex $x$ A. pegreffi, Pagellus bogaraveo, most larvae encapsulated in fish body cavity, mesenteries, on the ouside of the viscera, Hermida et al. (2012b).

Anisakis sp., Aphanopus carbo, Helicolenus dactylopterus, digestive tract, surface of viscera, Cruz et al. (2009), Santos et al. (2009), Sequeira et al. (2010).

Contracaecum sp., Pagellus bogaraveo, most larvae encapsulated in fish body cavity, mesenteries, on the ouside of the viscera, Hermida et al. (2012b).

\section{Crustacea}

Argulus foliaceus Linnaeus, 1758, Perca fluviatilis, Oncorhynchus mykiss, Cyprinus carpio, Esox lucius, tegument, Menezes et al. (1990a) (freshwater).
Argulus sp., Pagellus bogaraveo, pectoral fin, Hermida et al. (2013a, b).

Aega antillensis Schioedte \& Meinert, 1879 [=Aegaphelles antillensis (Schioedte \& Meinert, 1879)], Pagellus bogaraveo, gills, Hermida et al. (2013a, b).

Gnathia sp., Pagellus bogaraveo, mouth, oesophagous, gills, gill chamber, tegument, fins, Hermida et al. (2013a, b).

Hatschekia pagellibogneravei (Hesse, 1878), Pagellus bogaraveo, gills, Hermida et al. (2013a, b).

Rocinela danmoniensis Leach, 1818, Pagellus bogaraveo, tegument, Hermida et al. (2013a, b).

\section{Parasites of farmed fish}

Flagellata

Amyloodinium ocellatum (Brown, 1931), Coris julis, Diplodus puntazzo, D. sargus sargus (=D. sargus), D. vulgaris, Sparus aurata, Solea senegalensis, gills, Menezes (1992), Pereira et al. (2011), Soares et al. (2011).

Hexamita intestinalis Dujardin, 1841, Oncorhynchus mykiss, intestine, Carvalho Varela et al. (1981).

Ichthyobodo sp., Anguilla anguilla, Coris julis, Salmo trutta fario, tegument, Cruz e Silva et al. (1986), Menezes (1992), Saraiva et al. (1998).

\section{Apicomplexa}

Eimeria truttae (Léger \& Hesse, 1919) Stankovitch, 1924, Oncorhynchus mykiss, gut mucosa, Carvalho Varela et al. (1981). Eimeria sp., Anguilla anguilla, Coris julis, gut mucosa, Cruz e Silva et al. (1986), Menezes (1992).

\section{Ciliophora}

Ichthyophthirius multifiliis Fouquet, 1876, Anguilla anguilla, Oncorhynchus mykiss, gills, tegument, fins, Grazina Freitas \& Martins (1983), Ventura \& Paperna (1985), Cruz e Silva et al. (1986).

Trichodina anguillae Ergens, 1960, Anguilla anguilla, gills, Carvalho Varela et al. (1981).

T. truttae Müller, 1937, Oncorhynchus mykiss, gills, Carvalho Varela et al. (1981).

Trichodina sp., Coris julis, Sparus aurata, gills, skin, Cruz e Silva et al. (1977), Menezes (1992).

\section{Myxozoa}

Ceratomyxa auratae Rocha, Casal, Rangel, Castro, Severino, Azevedo \& Santos, 2015, Sparus aurata, gall bladder, Rocha et al. (2015a).

Ceratomyxa sp., Sparus aurata, gall bladder, Rocha et al. (2015b).

Myxidium giardi Cépède, 1906, Anguilla anguilla, skin, gills cartilage, liver, spleen, kidney, urinary bladder, intestine, Ventura \& Paperna (1984), Cruz e Silva et al. (1986). 
Ortholinea auratae Rangel, Rocha, Borkhanuddin, Cehh, Castro, Casal, Azevedo, Severino, Székely, Santos, 2014, Sparus aurata, urinary bladder, Rangel et al. (2014).

Zschokkella auratis Rocha, Casal, Rangel, Severino, Castro, Azevedo, Santos, 2013, Sparus aurata, gall bladder, Rocha et al. (2013).

\section{Monogenea}

Dactylogyrus sp., Anguilla anguilla, tegument, Cruz e Silva et al. (1986).

Diplectanum aequans (Wagener, 1857), Dicentrarchus labrax, gills, Saraiva et al. (2015a, b).

Gyrodactylus elegans Nordmann, 1832, Solea solea, gills, Carvalho Varela et al. (1981).

Gyrodactylus sp., Oncorhynchus mykiss, Salmo trutta, skin, Eiras (1999).

Lamellodiscus ignoratus Palombi, 1943, Sparus aurata, gills, skin, Cruz e Silva et al. (1997).

Lamellodiscus sp., Coris julis, gills, Menezes (1992).

Microcotyle sp., Coris julis, Sparus aurata, gills, skin, Menezes (1992), Cruz e Silva et al. (1997).

\section{Cestoda}

Bothriocephalus sp., Pleuronectes platessa, intestine, Carvalho Varela et al. (1981).

\section{Hirudinea}

Platybdella soleae (Grassé, 1959), Pegusa lascaris (=Solea lascaris), S. senegalensis, Solea solea (=S. vulgaris), skin, Barahona-Fernandes \& Dinis (1992).

\section{Nematoda}

Contracaecum aduncum, (Rudolphi, 1802) Railliet \& Henry, 1912, Pleuronectes platessa, intestine, Carvalho Varela et al. (1981).

Cucullanus minutus (Rudolphi, 1819) Tornquist, 1931, Solea solea, intestine, Carvalho Varela et al. (1981).

Raphidascaris sp., Mugil cephalus, intestine, Carvalho Varela et al. (1981).

\section{Crustacea}

Caligus minimus Otto, 1821, Dicentrarchus labrax, gills, Saraiva et al. (2015a, b).

\section{Discussion}

With regard to marine fish, 180 different parasites have been detected in 74 host species in mainland Portugal, 61 parasites in 20 host species in Madeira, and 29 parasites in 3 host species in the Azores. Considering all the marine species common to the three regions, we find that parasites have been found only in 86 different hosts. As for freshwater fish, 52 parasite species were reported in mainland hosts, and one parasite species was observed infecting 5 freshwater hosts in the Azores. As far as we know, there are no reports of parasite species in freshwater fish in Madeira. On the other hand, 22 parasites have been observed in both marine and freshwater farmed fish.

It is evident that most of the reports from the three different "sampling sites" refer to hosts of major economic importance. This is not surprising. Most of the findings resulted from research projects funded by government agencies and, on a competitive basis, the study of important economic species is naturally favored. On the other hand, a large proportion of the findings were included in MSc and $\mathrm{PhD}$ studies, which also concerned hosts of high economic impact.

It is also obvious that, in general, the study of protistan parasites has been overlooked. Only 11 species in mainland waters, only one in Madeira, and none in the Azores have been reported. This was probably due to the sampling conditions of most of the fish, which were purchased from fishermen or collected at experimental fisheries, and the hosts were examined at a later date. These sampling conditions are not suitable for the search of protists, namely those that are ectoparasites. This limitation must be taken into account in future research, and whenever possible, the "neglected" protistan parasites must be included in research projects. On the other hand, the monogeneans, digeneans, cestodes, nematodes and crustaceans were generally the most prevalent parasites among the metazoan species.

Considering the parasites from mainland hosts, it is evident that the number of host species examined is very low relative to the number of hosts reported for the area. Moreover, most of the described parasites came from a small number of the 74 reported hosts. This means that research into fish parasites should be extended to other species, even if they are not economically relevant.

Some parasites deserve special attention for several reasons. First, some species are dangerous parasites in farming or aquarium conditions. That is the case particularly of the protists Amyloodinium ocellatum (SARAIVA et al., 2011) and Cryptocaryon irritans, which are responsible for numerous cases of high mortality rates at farms and aquaria (COLORNI \& BURGESS, 1997).

Several monogeneans and copepods that locate on the gills of the hosts are also detrimental to the hosts, especially in cases of high infection intensity, and may be important at farms.

Kudoa sp. (most probably K. thyrsites) was described in several hosts, causing a variable degree of post-mortem myoliquefaction of the host, thus rendering them unsuitable for consumption (CRUZ E SILVA \& GRAZINA FREITAS, 1984; GRAZINA FREITAS et al., 1986; GILMAN \& EIRAS, 1998; MENEZES et al. 1990b; CRUZ et al., 2003). Gilman \& Eiras (1998) showed that this parasite is responsible for the rejection of large quantities of fish by the canning industry. This type of infection, and infection with related species, is impossible to fight and may have serious economic consequences in the exploitation of wild stocks of some species (see MORAN et al., 1999).

Nematodes are another extremely important group of parasites, especially those with a zoonotic potential (Anisakis spp., 
Hysterothylacium spp. and Contracaecum spp.). This problem will be discussed further on.

In Madeira, 61 parasites were reported infecting 19 hosts. However, most of the parasites were found in only four host species, i.e., Trachurus picturatus, Aphanopus carbo, Pagellus bogaraveo and Scomber japonicus, all of them species of high economic value. Once again, it was easier to obtain funding to study these valuable species. Nematodes were the most prevalent parasites, followed by cestodes, monogenea and digenea. It should be noted that most of the findings on fish parasites in Madeira are mainly due to the work of Costa G. et al. (1996, 1998, 2000, 2003a, b, 2004a, b, 2007, 2009a, b, 2012, 2013a, b, 2014, 2016), and also of Hermida et al. (2008, 2012a, b, 2013a, b, 2014, 2015), Santos et al. (2009) and Oliva et al. (2008).

An interesting issue that was addressed was the use of parasites as biological tags for the discrimination of Aphanopus carbo fish stocks from mainland Portugal, Madeira and Azores. The results of this cooperative research involving several institutions suggested that 6 parasites (Tentacularia coryphaenae, Sphyriocephalus tergestinus, Campbelliella heteropoeciloacantha, Anisakis spp., Bolbosoma vasculosum and unidentified Acanthocephala larvae) can be used as biological tags to discriminate Aphanopus carbo stocks from Portugal (SANTOS et al., 2009). This study illustrates the usefulness of several institutions cooperating to solve an interesting biological problem of potential economic interest, and parasites can probably be used for similar purposes involving other fish species.

Surprisingly, the 29 parasite species reported for the Azores (excluding Argulus foliaceus described in freshwater fish) were detected in only three marine host species - Aphanopus carbo (21 parasites), Pagellus bogaraveo (6) and Helicolenus dactylopterus (2)Hermida et al. (2013a, b, 2014), Hermida et al. (2012a, b, 2013). Furthermore, the papers by Hermida and Hermida et al. resulted from her $\mathrm{PhD}$ studies on the parasites of $P$. bogaraveo. This means that research on fish parasites in the Azores is practically nonexistent, in spite of the great diversity of fishes, and the marked economic importance of the fish in Azorean waters. This is a research field that obviously needs the attention of researchers from the central and local Fisheries authorities and from the academic sector.

According to Almaça (1996), there are 36 freshwater fish species in Portugal, most of them belonging to the family Cyprinidae (19 species). Research on fish parasites has focused only on ten fish species, resulting in the detection of 51 different parasites, 26 of which are described in Anguilla anguilla. This does not necessarily mean that this eel is more infected than other host species, but simply reflects a greater research effort directed towards this species, possibly because the economic potential of this host is greater than that of others. However, in general, the economic importance of Portugal's freshwater fishes is very low, and professional fisheries are almost nonexistent, with a few exceptions in some reservoirs and in major rivers.

The number of parasites detected so far is very small and, interestingly, the myxozoans are the most abundant parasites reported considering the number of species, a result that is in large part due to the paper by Molnár et al. (2012) about the myzozoans of Luciobarbus bocagei. Some species that occur in the wild may be dangerous in the case of fish farming, namely eel farming (especially Ichthyophthirius multifliis, Pseudodactylogyrus anguillae,
P. bini, Anguillicola crassus and several species of crustaceans, namely Lernaea cyprinacea).

Most of the papers concerning freshwater fish were produced for academic purposes, and there are no major research projects on the parasites of freshwater fish. On the other hand, practically all the data pertaining to hosts were collected in the north of the country, mostly in the vicinity of Porto, and only fragmentary data are available about hosts from the country's center and southern regions. Clearly, an integrated study should be conducted comprising the diversity of parasites and their geographic distribution, and host-parasite relationships - this could easily be done given the small number of host species, the facility of sampling by electrofishing and net fishing, and the small size of the country. The costs involved would not be high, and a number of qualified researchers with broad experience in the field would ensure the success of such a project.

The importance of fish parasites in fish farming stems from to the injuries and mortality rates they can cause on farmed species. Fish farming is scantily developed in Portugal, and the number of parasites described in aquaculture facilities is very low, i.e., only 25 different species. Some of them are not particularly dangerous and do not require special care. However, some are very important for the fish farmer and may, in favorable conditions, pose a threat to exploitation if adequate measures are not taken in time. The abundance of those species requires continuous monitoring in order to deploy adequate disinfection procedures to keep infection rates at levels that do not interfere with the survival of the hosts.

Several parasites have been linked to abnormal mortality rates in fish farming in this country. The monogeneans Microcotyle sp., Lamellodiscus ignoratus and the ciliate Trichodina sp. caused mortality in Sparus aurata, mostly in association with poor environmental conditions involving the penetration of freshwater into marine aquaculture premises, which led to a decrease in the salinity of the water. At several trout farms, the monogenean Gyrodactylus sp. caused mortality in spring and summer due to the increase in water temperature. Yearly high mortality rates have been caused by Amyloodinium ocellatum in farmed Diplodus sargus, D. vulgaris, D. puntazzo and Solea senegalensis in spring and summer owing to high water temperatures. Ciliates belonging to the order Philateridae caused high mortality rates in Scophthalmus maximus (RAMOS et al., 2007).

The ciliate Icthyophthirius multifiliis is considered to be possibly the most important ectoparasite in freshwater fish farming, and has been reported in all the freshwater fish farms in the country, causing White Spot Disease. Despite the high mortality levels this ectoparasite may cause, monitoring of the fish and adequate prophylactic measures are usually the means to prevent high economic losses. However, several cases of mortality have been observed in Oncorhynchus mykiss (EIRAS, unpublished observations) and Anguilla anguilla, and stressed O. mykiss were reported by Ventura \& Paperna (1985). Another case of mortality was observed in the marine fish Coris julis due to infection by Amyloodinium ocellatum (MENEZES, 1992).

Some observations have revealed that parasites may adversely affect several organs of farmed fish, and this problem requires 
particular attention because of the lesions they produce. This is the case of Diplectanum aequans and Caligus minimus, which have been found to contribute to severe histological gill alterations in farmed Dicentrarchus labrax (SARAIVA et al., 2015ab), and of Myxidium giardi, which cause pathological lesions in the kidney of elvers (VENTURA \& PAPERNA, 1984).

It should be noted that Lepeophtheirus salmonis, the "salmon louse," has been detected in wild Salmo trutta in Minho catchment (BAO et al., 2016). The importance of this parasite, which causes heavy losses especially in net-cage farmed Salmo salar (COSTELLO, 2009; SKILBREI et al., 2013), is well known and must be taken into consideration in future farms of the host.

Overall, it can be stated that, despite the occurrence of some cases of mortality, the influence of parasites in Portuguese fish farming is negligible. This is due to the scanty development of fish farming in the country and to the action of the fish-farmers who monitor farm premises constantly in order to adopt the necessary measures to prevent parasite outbreaks. For a general overview of the influence of parasites on fish farms, see Eiras (1994) and Carvalho Varela (2005).

As stated earlier, a large number of hosts were infected by potentially zoonotic nematodes (Anisakis spp., Hysterothylacium spp. and Contracaecum spp.) at all the sampling sites. The problem of human infection by fish nematodes in Portugal was discussed by Carvalho Varela \& Cunha Ferreira (1984), Ramos (1998, 2011), Rombert (1993), Silva \& Eiras (2003), Nunes et al. (2003) and Bao et al. (2015). Human infection is acquired by ingesting raw or poorly cooked fish, and infections have different symptomatologies according to the nematodes ingested. The symptoms of infection are often difficult to recognize because they are similar to those of other diseases.

As far as the author knows, there are no notified cases of human infections by fish nematodes in Portugal. However, Nunes et al. (2003) found 8 individuals showing specific antibodies to Anisakis simplex in the bloodstream. It must be kept in mind that allergic reactions to Anisakis, including anaphylaxis, may occur even when properly cooked fish is ingested (AUDICANA et al., 2002). Portuguese dishes do not usually include raw fish. However, "sushi" and "sashimi" restaurants, which have become "fashionable," can be considered places that pose a probable risk factor for infection (RAMOS, 2011).

Our data indicate that infection of fish by zoonotic nematodes is frequent in Portuguese waters, and we agree with the opinion of the European Food Safety Authorithy, which considers that "All wild caught seawater and freshwater fish must be considered at risk of containing any viable parasites of human health concern if these products are to be eaten raw or almost raw" (EFSA, 2010). More particularly, "For wild-caught fish, no sea fishing grounds can be considered free of $A$. simplex" (EFSA, 2010). Furthermore, all fish species should be considered potentially dangerous and processed accordingly for human consumption. Therefore, some precautions should be adopted to prevent infection and we suggest following the recommendations of the US Food and Drug Administration for consumption of raw fish (US Food and Drug Administration, 2011).

\section{Conclusions}

1. The studies of fish parasites in Portugal (mainland, and the archipelagos of Madeira and Azores) have been developed mostly over the last three decades. A total of 180 parasite species of marine fish have been reported in the mainland coastal waters, 52 in Madeira, 29 in the Azores, 52 in freshwater fish in the mainland, and 22 in farmed fish. This means that the great majority of fish species have never been studied from the parasitological point of view.

2. Knowledge about parasitic protists is very scanty when it comes to metazoan parasites, and this is gap that must be filled.

3. Most of the studies on marine fish have involved only important commercial species. The parasite fauna in other species are mostly unknown.

4. Most of the studies on freshwater parasites have involved Anguilla anguilla, while the parasites of the majority of freshwater fish species have never been studied.

5.A number of nematodes with zoonotic potential are common parasites in marine fish in mainland coastal waters, Madeira and Azores. This fact must be taken into account when cooking fish, and the consumption of raw or undercooked fish should be avoided.

\section{Acknowledgements}

This paper was partially funded by the "European Regional Development Fund" (ERDF) through the Operational Competitiveness Programme and National Funds - COMPETE via FCT - Fundação para a Ciência e Tecnologia, under the project "Pest-C/MAR/LA0015/2013". The author is indebted to several colleagues who assisted him in the bibliographical research: Aurélia Saraiva, Cristina Cruz, Graça Costa, Ken MacKenzie, Margarida Hermida, Maria João Santos. He is also grateful to the journal Revista Brasileira de Parasitologia Veterinária - RBPV for offering to publish this review.

\section{References}

Almaça C. Peixes dos rios de Portugal. Lisboa: Inapa Press; 1996.

Audicana MT, Ansotegui IJ, Corres LE, Kennedy MW. Anisakis simplex: dangerous - dead and alive? Trends Parasitol 2002; 18(1): 20-25. http:// dx.doi.org/10.1016/S1471-4922(01)02152-3. PMid:11850010.

Azevedo C, Lorn J, Corral L. Ultrastructural aspects of Myxidium giardi (Myxozoa, Myxosporea) parasite of the european eel Anguilla anguilla. Dis Aquat Organ 1989; 6(1): 55-61. http://dx.doi.org/10.3354/dao006055.

Bao M, Costal D, Garci ME, Pascual S, Hastie LC. Sea lice (Lepeophtheirus salmonis) and anchor worms (Lernaea cyprinacea) found on sea trout (Salmo trutta) in the River Minho catchment, an important area for conservation in NW Spain. Aquatic Conserv: Mar Freshw Ecosyst 2016; 26(2): 386-391. http://dx.doi.org/10.1002/aqc.2572. 
Bao M, Mota M, Nachón DJ, Antunes C, Cobo F, Garci ME, et al. Anisakis infection in allis shad, Alosa alosa (Linnaeus, 1758), and twaite shad, Alosa fallax (Lacépède, 1803), from Western Iberian Peninsula Rivers: zoonotic and ecological implications. Parasitol Res 2015; 114(6): 2143 2154. http://dx.doi.org/10.1007/s00436-015-4403-5. PMid:25810220.

Barahona-Fernandes MH, Dinis MT. Leech control (Platybdella solea, Hirudinae) on flatfishes in an aquaculture unit. Publ Inst Zool. Dr. A. Nobre 1992; 226: 1-4.

Borges R, Ré P, Azevedo C. Ichthyodinium chabelardi (Hollande e Cachon 1952), dinoflagelado parasita dos ovos de sardinha. Ciênc Biol Ecol Syst 1996; 16(1-2): 245-258.

Buhrnheim U, Gomes DC, Varela MC. Alguns trematódeos monogenéticos da família Capsalidae (Blaird), 1853, em peixes do Oceano Atlântico - costa continental portuguesa e costa do norte da África. Mem Inst Oswaldo Cruz 1973; 71(3): 227-239. http://dx.doi.org/10.1590/S007402761973000200003.

Candeias A. Rebelula edwardsii (Kolliker) on a Coelorhynchus coelorhynchus (Risso) from the coast of Portugal. Notas e Estudos Inst Biol Marit 1952a; 1: 15 .

Candeias A. On Peroderma cylindricum (Heller) parasite of Sardina pilchardus (Walb.) from the Atlantic. Notas e Estudos Inst Biol Marit 1952b; 4: 10.

Candeias A. Peniculus fistula Nordm. on two new hosts Trachurus trachurus (L.) and Trachurus picturatus (Bowd). Notas e Estudos Inst Biol Marit 1955; 8: 16.

Cardoso C, Lourenço H, Costa S, Gonçalves S, Nunes ML. Survey into the seafood consumption preferences and patterns in the Portuguese population: education, age, and health variability. J Food Prod Mark 2016; 22(4): 421-435. http://dx.doi.org/10.1080/10454446.2014.949982.

Cardoso EM, Saraiva A. Distribution and seasonal occurrence of Anguillicola crassus (Nematoda: Dracunculoidea) in the European Eel Anguilla anguilla L. from rivers of North Portugal. Bull Eur Assoc Fish Pathol 1998; 18(4): 136-139.

Carneiro M, Martins R, Landi M, Costa FO. Updated checklist of marine fishes (Chordata: Craniata) from Portugal and the proposed extension of the Portuguese continental shelf. Eur J Taxon 2014; 73(1): 1-73. http:// dx.doi.org/10.5852/ejt.2014.73.

Carvalho Varela M. Perspectivas gerais da helmintologia ictiológica. Bol Pecuário 1975; 43: 37-83.

Carvalho Varela M. Parasitas e parasitoses em piscicultura. Lisboa: Ordem dos Médicos Veterinários; 2005.

Carvalho Varela M, Cunha-Ferreira V. Larva Migrans visceral por Anisakis e outros ascarídeos: helmintozoonoses potenciais por consumo de peixes marinhos em Portugal. Rev Port Ciênc Vet 1984; 89(472): 299-309.

Carvalho Varela M, Cunha Ferreira V. Helminth parasites of the common sole, Solea solea, and the senegalese sole, Solea senegalensis on the Portuguese continental coast. Aquaculture 1987; 67(1-2): 135-138. http://dx.doi. org/10.1016/0044-8486(87)90018-4.

Carvalho Varela M, Cunha-Ferreira V, Silva MPC, Monteiro MT, GrazinaFreitas MS. Parasites and parasitosis in fish culture in Portugal. $J$ World Maricul Soc 1981; 12(2): 9-14. http://dx.doi.org/10.1111/j.1749-7345.1981. tb00271.x.

Casal G, Costa G, Azevedo C. Ultrastructural description of Ceratomyxa tenuispora (Myxozoa) a parasite of the marine fish Aphanopus carbo (Trichiuridae), from the Atlantic coast of Madeira Island (Portugal). Folia
Parasitol (Praha) 2007; 54(3): 165-171. http://dx.doi.org/10.14411/ fp.2007.023. PMid:19245187.

Castro S, Santos MJ. Metazoan ectoparasites of Atlantic mackerel, Scomber scombrus, (Teleostei, Scombridae): macro- and microhabitat distribution. Parasitol Res 2013; 112(10): 3579-3586. http://dx.doi.org/10.1007/ s00436-013-3543-8. PMid:23912195.

Cavaleiro F, Pina S, Russell-Pinto F, Rodrigues P, Formigo NE, Gibson DI, et al. Morphology, ultrastructure, genetics, and morphometrics of Diplostomum sp. (Digenea: Diplostomidae) metacercariae infecting the European flounder, Platichthys flesus (L.) (Teleostei: Pleuronectidae), off the northwest coast of Portugal. Parasitol Res 2012; 110(1): 81-93. http:// dx.doi.org/10.1007/s00436-011-2453-x. PMid:21626424.

Cavaleiro F, Santos MJ, Ho J. Caligus musaicus n. sp. (Copepoda, Caligidae) parasitic on the European flounder, Platichthys flesus (Linnaeus) off Portugal. Crustaceana 2010; 83(4): 457-464. http://dx.doi. org/101163/001121610X489359.

Cavaleiro F, Santos MJ. Seasonality of metazoan ectoparasites in marine European flounder Platichthys flesus (Teleostei: Pleuronectidae). Parasitology 2009; 136(8): 855-865. http://dx.doi.org/10.1017/S003118200900626X. PMid:19470193.

Cavaleiro F, Santos MJ. Site selection of Acanthochondria cornuta (Copepoda: Chondracanthidae) in Platichthys flesus (Teleostei: Pleuronectidae). Parasitology 2011; 138(8): 1061-1067. http://dx.doi.org/10.1017/ S0031182011000606. PMid:21733262.

Cavaleiro F, Santos MJ. Survey of the metazoan ectoparasites of the European Flounder Platichthys flesus (Linnaeus, 1758) along the NorthCentral Portuguese coast. J Parasitol 2007; 93(5): 1218-1222. http:// dx.doi.org/10.1645/GE-1071R1.1. PMid:18163362.

Chubb JC, Eiras JC, Saraiva A. Khawia baltica (Cestoidea: Caryophyllidea) from Barbus barbus bocagei (Linnaeus, 1758) from some rivers in northern Portugal. Folia Parasitol (Praha) 1997; 44(2): 131-138.

Colorni A, Burgess P. Cryptocaryon irritans Brown 1951, the cause of "white spot disease" in marine fish: an update. Aquarium Sci Conserv 1997; 1(4): 217-238. http://dx.doi.org/10.1023/A:1018360323287.

Costa FO, Landi M, Martins R, Costa MH, Costa ME, Carneiro M, et al. A ranking system for reference libraries of DNA Barcodes: application to marine fish species from Portugal. PLoS One 2012; 7(4): e35858. http:// dx.doi.org/10.1371/journal.pone.0035858. PMid:22558244.

Costa G, Biscoito M. Helminth parasites of some coastal fishes from Madeira, Portugal. Bull Eur Assoc Fish Pathol 2003; 23(6): 281-286.

Costa G, Chada T, Melo-Moreira E, Cavallero S, D’Amélio S. Endohelminth parasites of the leafscale gulper shark, Centrophorus squamosus (Bonnaterre, 1788) (Squaliformes: Centrophoridae) off Madeira Archipelago. Acta Parasitol 2014; 59(2): 316-322. http://dx.doi.org/10.2478/s11686-0140247-x. PMid:24827105.

Costa G, Chubb JC, Veltkamp CJ. Cystacanths of Bolbosoma vasculosum in the black scabbard fish Aphanopus carbo, oceanic horse mackerel Trachurus picturatus and common dolphin Delphinus delphis from Madeira, Portugal.J Helminthol 2000; 74(2): 113-120. http://dx.doi.org/10.1017/ S022149X00000159. PMid:10881281.

Costa G, Eiras JC, Chubb J, MacKenzie K, Berland B. Parasites of the black scabbard fish, Aphanopus carbo Lowe, 1839 from Madeira. Bull Eur Assoc Fish Pathol 1996; 16(1): 13-16.

Costa G, Freitas N, Dellinger TH, MacKenzie K. Gill monogeneans of the chub mackerel, Scomber japonicus from Madeiran waters of the 
Atlantic Ocean, Portugal. J Helminthol 2007; 81(1): 33-38. http://dx.doi. org/10.1017/S0022149X07207333. PMid:17381864.

Costa G, Freitas N, Pinheiro de Carvalho MAA, Melo-Moreira E, Rigby MC. Oncophora melanocephala (Nematoda, Camallanidae) from the chub mackerel, Scomber japonicus (Teleostei, Scombridae), caught off Madeira Island (Portugal). Acta Parasitol 2009a; 54(3): 253-256. http://dx.doi. org/10.2478/s11686-009-0032-4.

Costa G, Santos MJ, Costa L, Biscoito M, Pinheiro de Carvalho MAAP, Melo-Moreira E. Helminth parasites from the stomach of conger eel, Conger conger, from Madeira Island, Atlantic Ocean. J Parasitol 2009b; 95(4): 1013-1015. http://dx.doi.org/10.1645/GE-1760.1. PMid:20050008.

Costa G, Khadem M, Dellinger T, Biscoito M, Melo-Moreira E. Larval cestodes infecting the deep-water fish Cataetyx laticeps (Pisces: Bythitidae) from Madeira Archipelago, Atlantic Ocean. Acta Parasitol 2016; 61(1): 187-190. http://dx.doi.org/10.1515/ap-2015-0025. PMid:27149705.

Costa G, Khadem M, Silva S, Moreira EM, D’Amélio S. Endohelminth parasites of the blacktail comber Serranus atricauda (Pisces: Serranidae), from Madeira Archipelago (Atlantic Ocean). Dis Aquat Organ 2013a; 103(1): 55-64. http://dx.doi.org/10.3354/dao02564. PMid:23482385.

Costa G, Santamaria MTG, Vasconcelos J, Perera CB, Melo-Moreira E. Endoparasites of Trachurus picturatus (Pisces: Carangidae) from the Madeira and Canary Islands: selecting parasites for use as tags. Sci Mar 2013b; 77(1): 61-68. http://dx.doi.org/10.3989/scimar.03707.07A.

Costa G, Lom J, Andrade C, Barradas R. First report of Ceratomyxa sparusaurati (Protozoa: Myxosporea) and the occurrence of epitheliocystis in cultured sea bream, Sparus aurata L. from Madeira. Bull Eur Assoc Fish Pathol 1998; 18(5): 165-167.

Costa G, Madeira A, Pontes T, D’Amélio S. Anisakid nematodes of the blackspot seabream, Pagellus bogaraveo, from Madeiran waters, Portugal. Acta Parasitol 2004a; 49(2): 156-151.

Costa G, Pontes T, Rego AA. Prevalence, intensity and abundance of Rhadinorhynchus pristis (Acanthocephala, Rhadinorhynchidae) in chub mackerel, Scomber japonicus (Pisces, Scombridae) from Madeira Island. Acta Parasitol 2004b; 49(1): 41-44.

Costa G, Melo-Moreira E, Pinheiro de Carvalho MAA. Helminth parasites of the oceanic horse mackerel Trachurus picturatus Bowdich 1825 (Pisces: Carangidae) from Madeira Island, Atlantic Ocean, Portugal. J Helminthol 2012; 86(3): 368-372. http://dx.doi.org/10.1017/S0022149X11000502. PMid:21875447.

Costa G, Pontes T, Mattiucci S, D'Amélio S. The occurrence and infection dynamics of Anisakis larvae in the black-scabbard fish, Aphanopus carbo, chub mackerel, Scomber japonicus, and oceanic horse mackerel, Trachurus picturatus from Madeira, Portugal. J Helminthol 2003a; 77(2): 163-166. http://dx.doi.org/10.1079/JOH2002156. PMid:12756070.

Costa G, Veltkamp CJ, Chubb JC. Larval trypanorhynchs (Platyhelminthes: Eucestoda: Trypanorhyncha) from black-scabbard fish, Aphanopus carbo and oceanic horse mackerel, Trachurus picturatus in Madeira (Portugal). Parasite 2003b; 10(4): 325-331. http://dx.doi.org/10.1051/ parasite/2003104325. PMid:14710629.

Costa JL, Marques JF, Alves J, Gamito R, Fonseca VS, Gonçalves CI, et al. Is parasitism in fish a good metric to assess ecological water quality in transitional waters? What can be learned from two estuarine resident species? Ecol Indic 2012; 19: 154-160. http://dx.doi.org/10.1016/j. ecolind.2011.08.025.
Costello MJ. The global economic cost of sea lice to the salmonid farming industry. J Fish Dis 2009; 32(1): 115-118. http://dx.doi.org/10.1111/ j.1365-2761.2008.01011.x. PMid:19245636.

Cruz C, Barbosa C, Saraiva A. Distribution of larval anisakids in blue whiting off Portuguese fish market. Helminthologia 2007; 44(1): 21-24. http://dx.doi.org/10.2478/s11687-006-0051-8.

Cruz C, Davies A. Some observations on Babesiosoma bettencourti (França, 1908) n. comb. (syns. Haemogregarina bettencourti França, 1908; Desseria bettencourti Siddall, 1995) from eels, Anguilla anguilla L., in Portugal. J Fish Dis 1998; 21(6): 443-448. http://dx.doi.org/10.1046/j.13652761.1998.00131.x.

Cruz C, Eiras JC. Prevalence of Trypanosoma granulosum in Anguilla anguilla in Portugal. Bull Eur Assoc Fish Pathol 1997; 17(3-4): 126-128.

Cruz C, Ferreira S, Saraiva AM. Ocorrência de Myxobolus cyprini em Chondrostoma polylepis em Portugal. Acta Parasitol Port 1998; 5(2): 63-66.

Cruz C, Saraiva A, Ferreira S. Preliminary observations on Myxobolus sp. from Cyprinid fishes in Portugal. Bull Eur Assoc Fish Pathol 2000, 20(2): 65-69.

Cruz C, Saraiva A, Santos MJ, Eiras JC, Ventura C, Soares JP, et al. Anisakis (Nematoda, Anisakidae) infestation in Aphanopus carbo (Osteichthyes, Trichiuridae) from Portuguese waters. Sci Mar 2009; 73(S2): 115-120. http://dx.doi.org/10.3989/scimar.2009.73s2115.

Cruz C, Vaz A, Saraiva A. Occurrence of Kudoa sp. (Myxozoa) in Trachurus trachurus L. (Osteichthyes) in Portugal. Parasite 2003; 10(2): 165-167. http://dx.doi.org/10.1051/parasite/2003102165. PMid:12847925.

Cruz C, Vaz A, Saraiva A. Larval anisakids from horse mackerel in Portugal. Helminthologia 2005; 42(1): 3-7.

Cruz e Silva MP, Grazina-Freitas MS. Kudoa sp. em músculo de pescada (Merluccius merluccius L.). Rep Trab LNIV 1984; 16: 151-154.

Cruz e Silva P, Grazina-Freitas MS, Carvalho Varela M. First report of Anguillicola crassus in the european eel in Portugal. Bull Eur Assoc Fish Pathol 1992; 12(5): 154-156.

Cruz e Silva MP, Grazina-Freitas MS, Orge ML. Co-infection by monogenetic trematodes of the genus Microcotyle V. Beneden \& Hesse 1863, Lamellodiscus ignoratus Palombi, 1943, the protozoan Trichodina sp. Ehrenberg, 1838 and the presence of epitheliocystis, Vibrio algynoliticus and V. vulnificus in cultured seabream (Sparus aurata L.) in Portugal. Bull Eur Assoc Fish Pathol 1997; 17(2): 40-42.

Cruz e Silva MP, Ventura MT, Grazina-Freitas MS. Binómio patologia/ maneio numa anguilicultura portuguesa. Rep Trab LNIV 1986; 18: 41-47.

Davies AJ, Eiras JC, Austin RTE. Investigations into the transmission of Haemogregarina bigemina Laveran \& Mesnil, 1901 (Apicomplexa: Adeleorina) between intertidal fishes in Portugal. J Fish Dis 1994; 17(3): 283-289. http://dx.doi.org/10.1111/j.1365-2761.1994.tb00223.x.

Dias PJ, Eiras JC, Davies AJ. A six-month duration, follow-up study of Haemogregarina bigemina at Foz do Douro, North Portugal, between ten and twenty years on. Bull Eur Assoc Fish Pathol 2005; 25(4): 161-165.

Duarte N, Rosa N, Santos MJ, Rebelo J. Estudo de ectoparasitas das brânquias de robalo (Dicentrarchus labrax L.) da Ria de Aveiro. Rev Biol 2000; 18(2): 59-68.

Durieux EDH, Marques JF, Sasal P, Bégout M-L, Cabral HN. Comparison of Solea solea macroparasites between two nursery: continental shelf systems in the Bay of Biscay and the Portuguese coast. J Fish Biol 2007; 70(6): 1921-1930. http://dx.doi.org/10.1111/j.1095-8649.2007.01460.x. 
EFSA Panel on Biological Hazards (BIOHAZ) - EFSA. Scientific Opinion on risk assessment of parasites in fishery products. EFSA Journal 2010; 8(4): 1543. http://dx.doi.org/10.2903/j.efsa.2010.1543.

Eiras JC. Unusual attachment sites of the parasitic copepod Lernaeocera lusci (Basset-Smith, 1896) upon Trisopterus luscus L. Bull Eur Assoc Fish Pathol 1984; 4(2): 28-29.

Eiras JC. Some aspects of the infection of bib, Trisopterus luscus (L.), by the parasitic copepod Lernaeocera lusci (Basset-Smith, 1896) in Portuguese waters. J Fish Biol 1986; 28(2): 141-145. http://dx.doi. org/10.1111/j.1095-8649.1986.tb05151.x.

Eiras JC. Occurrence of Haemogregarina bigemina (Protozoa: Apicomplexa) in Blennius pholis (Pisces: Blenniidae) along the Portuguese west coast. J Fish Biol 1987a; 30(5): 597-603. http://dx.doi.org/10.1111/j.1095-8649.1987. tb05787.x.

Eiras JC. Detection of Haemogregarina bigemina (Protozoa: Apicomplexa) infection in fish erythrocytes by phase-contrast microscopy. J Fish Biol 1987b; 31(2): 291-292. http://dx.doi.org/10.1111/j.1095-8649.1987. tb05233.x.

Eiras JC. Trypanosoma granulosum Laveran and Mesnil, 1902 (Protozoa, Kinetoplastida) infecting Anguilla anguilla L. in Portugal. Ciênc Biol Ecol Syst 1988; 8(1-2): 29-36.

Eiras JC. Indice bibliográfico de trabalhos de autores portugueses no domínio da ictiopatologia. Rev Univ Coimbra 1990a; 25: 163-175.

Eiras JC. Observations on erythrocyte abnormalities in fish. Bull Eur Assoc Fish Pathol 1990b; 10(3): 64-68.

Eiras JC. Elementos de Ictioparasitologia. Porto: Fundação Eng. António de Almeida; 1994.

Eiras JC. Parasitas de peixes de Portugal. Acta Parasitol Port 1998; 5(2): 99-105.

Eiras JC. Ocorrência de Gyrodactylus spp. (Monogenea, Gyrodactylidae) em Portugal e sua importância. Acta Parasitol Port 1999; 2(1): 71-73.

Eiras JC, Davies AJ. Haemogregarina bigemina Laveran \& Mesnil, 1901 (Protozoa, Apicomplexa) from Blennius pholis L. (Osteichthyes, Blenniidae): an investigation of seasonality in Portugal. J Fish Dis 1991; 14(6): 683-687. http://dx.doi.org/10.1111/j.1365-2761.1991.tb00627.x.

Eiras JC, Reichenbach-Klinke H-H. Nematoden als ursache von Darmknoten bei Süsswassernfischen. Fisch und Umwelt 1982; 11(1): 47-55.

Eiras JC, Santos PJ. The morphology and morphometry of Lernaeocera lusci Basset-Smith, 1896 (Copepoda, Pennellidae). Publ Inst Zool Dr A Nobre 1990; 219: 1-10.

Eiras JC, Santos PJ. New host record Lepidorhombus boscii (Risso, 1810), for Lernaeocera lusci (Bassett-Smith, 1896) (Copepoda, Pennellidae). Crustaceana 1992; 63(1): 91-92. http://dx.doi.org/10.1163/156854092X00316.

Eiras JC, Saraiva A, Cruz C. A note on the portuguese situation concerning some notifiable fish diseases. Aquaculture 1987; 67(3-4): 279-281. http:// dx.doi.org/10.1016/0044-8486(87)90212-2.

Esteves A, Seixas F, Carvalho S, Nazário N, Mendes M, Martins C. Huffmanela sp. (Nematoda: Trichosomoididae) muscular parasite from Trisopterus luscus captured off the Portuguese coast. Dis Aquat Organ 2009; 84(3): 251-255. http://dx.doi.org/10.3354/dao02048. PMid:19565703.

França C. Le trypanosome de l'anguille (T. granulosum) Laveran and Mesnil. Arch Inst Bacteriol Câmara Pestana 1907; 2: 113-121.
Francisco CJ, Almeida A, Castro AM, Santos MJ. Development of a PCR-RFLP marker to genetically distinguish Prosorhynchus crucibulum and Prosorhynchus aculeatus. Parasitol Int 2010; 59(1): 40-43. http:// dx.doi.org/10.1016/j.parint.2009.09.004. PMid:19818870.

Francisco CJ, Hermida MA, Santos MJ. Prosorhynchus crucibulum (Digenea: Bucephalidae) miracidium morphology and its passive transmission pattern. Parasite 2012; 19(3): 277-280. http://dx.doi.org/10.1051/ parasite/2012193277. PMid:22910671.

Freitas MV, Marques JF, Cabral HN. Parasitological diversity of the common goby, Pomastochistus microps (Krøyer, 1838), in estuarine systems along the Portuguese coast. J Appl Ichthyology 2009; 25(2): 168-172. http://dx.doi.org/10.1111/j.1439-0426.2008.01205.x.

Froese R, Pauley D, editors. FishBase: Worl Wide Web electronic publications: version 01/2016 [online] 2016. [cited 2016 Jun 01] Available from: www.fishbase.org

Gestal C, Azevedo C. Ultrastructure of Goussia cruciata (Apicomplexa: Coccidia) infecting the liver of horse mackerel, Trachurus trachurus (L.), from Ibero-Atlantic waters. J Fish Dis 2005; 28(3): 125-132. http://dx.doi. org/10.1111/j.1365-2761.2005.00611.x. PMid:15752272.

Gestal C, Azevedo C. Ultrastructural aspects of hepatic coccidiosis caused by Goussia lusca n. sp. (Apicomplexa: Coccidia) infecting Trisopterus luscus (Gadidae) from the NE Atlantic Ocean. Dis Aquat Organ 2006; 71(1): 25-31. http://dx.doi.org/10.3354/dao071025. PMid:16921998.

Gibson DI, Costa G. Helminth parasites of Madeiran rockpool fishes, with a redescription of Schikhobalotrema longivesiculatum Orecchia \& Paggi, 1975 (Digenea: Haplosplanchnidae), and some comments on their zoogeographical relationships. Syst Parasitol 1997; 38(1): 73-79. http://dx.doi.org/10.1023/A:1005833826648.

Gilman MM, Eiras JC. Kudoa sp. (Myxosporea: Multivalvulida) infecting Sardina pilchardus (Walb., 1792) off the Portuguese coast. Res Rev Parasitol 1998; 58(2): 135-137.

Grazina-Freitas MS, Cruz e Silva MP, Ventura MT. Patologia de peixes marinhos. Casos estudados no Laboratório Nacional de Investigação Veterinária. Rep Trab LNIV 1986; 18: 61-66.

Grazina-Freitas MS, Martins MS. A ictioftiriose em trutas arco-íris de cultura. Rep Trab LNIV 1983; 15: 117-122.

Hermida M, Cruz C, Saraiva A. Distribution of Hatschekia pagellibogneravei (Copepoda: Hatschekiidae) on the gills of Pagellus bogaraveo (Teleostei: Sparidae) from Madeira, Portugal. Folia Parasitol (Praha) 2012a; 59(2): 148-152. http://dx.doi.org/10.14411/fp.2012.020. PMid:22779115.

Hermida M, Mota R, Pacheco CC, Santos CL, Cruz C, Saraiva A, et al. Infection levels and diversity of anisakid nematodes in blackspot seabream, Pagellus bogaraveo, from Portuguese waters. Parasitol Res 2012b; 110(5): 1919-1928. http://dx.doi.org/10.1007/s00436-011-2718-4. PMid:22139402.

Hermida M, Cruz C, Saraiva A. Ectoparasites of the blackspot seabream Pagellus bogaraveo (Teleostei: Sparidae) from Portuguese waters of the north-east Atlantic. J Mar Biol Assoc U K2013a; 93(2): 503-510. http:// dx.doi.org/10.1017/S0025315412000057.

Hermida M, Cruz C, Saraiva A. Parasites as biological tags for stock identification of blackspot seabream, Pagellus bogaraveo, in Portuguese northeast Atlantic waters. Sci Mar 2013b; 77(4): 607-615. http://dx.doi. org/10.3989/scimar.03859.17A.

Hermida M, Cruz C, Saraiva A. Gastrointestinal helminth communities of the blackspot seabream Pagellus bogaraveo (Teleostei: Sparidae) from Portuguese north-east Atlantic waters. J Helminthol 2014; 88(2): 129138. http://dx.doi.org/10.1017/S0022149X1200079X. PMid:23182041. 
Hermida M, Pereira A, Correia AT, Cruz C, Saraiva A. Metazoan parasites of blue jack mackerel Trachurus picturatus (Perciformes: Carangidae) from Portuguese mainland waters. J Helminthol 2015; 90(4): 410-416. http:// dx.doi.org/10.1017/S0022149X15000504. PMid:26121918.

Hermida M, Saraiva A, Cruz C. Metazoan parasite community of a European eel (Anguilla anguilla) population from an estuary in Portugal. Bull Eur Assoc Fish Pathol 2008; 28(1): 35-40.

Kearn GC, Vasconcelos ME. Preliminary list of monogenean parasites of Portuguese marine fishes, with a note on Euplocotyle minima Taglioni, 1912. Bol Inst Nac Invest Pescas 1979; 1(1): 25-36.

Machado Cruz JA. Lernaeocera caparti sp. nov.: copépode parasite de Merluccius merluccius (Linné). Publ Inst Zool Dr A Nobre 1959a; 64: 1-12.

Machado Cruz JA. Hepatoxylon trichiuri (Holten, 1802): nota sobre o seu aparecimento na costa de Portugal. Publ Inst Zool Dr A Nobre 1959b; 65: 1-7.

MacKenzie K, Campbell N, Mattiucci S, Ramos P, Pinto AL, Abaunza P. Parasites as biological tags for stock identification of Atlantic horse mackerel Trachurus trachurus L. Fish Res 2008; 89(2): 136-145. http:// dx.doi.org/10.1016/j.fishres.2007.09.031.

Marques JF, Cabral HN. Effects of sample size on fish parasite prevalence, mean abundance and mean intensity estimates. J Appl Ichthyology 2007; 23(2): 158-162. http://dx.doi.org/10.1111/j.1439-0426.2006.00823.x.

Marques JF, Cabral HN, Busi M, D’Amelio S. Molecular identification of Anisakis species from Pleuronectiformes off the Portuguese coast. J Helminthol 2006a; 80(1): 47-51. http://dx.doi.org/10.1079/JOH2005325. PMid:16469172.

Marques JF, Santos MJ, Cabral HN. Soleidae macroparasites along the Portuguese coast: latitudinal variation and host-parasite associations. Mar Biol 2006b; 150(2): 285-298. http://dx.doi.org/10.1007/s00227006-0339-8.

Marques JF, Teixeira CM, Cabral HN. Differentiation of commercially important flatfish populations along the Portuguese coast: evidence from morphology and parasitology. Fish Res 2006c; 81(2-3): 293-305. http:// dx.doi.org/10.1016/j.fishres.2006.05.021.

Marques JF, Santos MJ, Cabral HN. Zoogeographical patterns of flatfish (Pleuronectiformes) parasites in the Northeast Atlantic and the importance of the Portuguese coast as a transitional area. Sci Mar 2009; 73(3): 461471. http://dx.doi.org/10.3989/scimar.2009.73n3461.

Marques JF, Santos MJ, Cabral HN. Aggregation patterns of macroendoparasites in phylogenetically related fish hosts. Parasitology 2010; 137(11): 16711680. http://dx.doi.org/10.1017/S0031182010000491. PMid:20500921.

Marques JF, Santos MJ, Cabral HN, Palm HW. First record of Progrillotia dasyatidis Beveridge Neifar and Euzet, 2004 (Cestoda: Trypanorhyncha) plerocerci from Teleost fishes off the Portuguese coast, with a description of the surface morphology. Parasitol Res 2005a; 96(4): 206-211. http:// dx.doi.org/10.1007/s00436-005-1341-7. PMid:15864648.

Marques JF, Santos MJ, Costa JL, Costa MJ, Cabral HN. Metazoan parasites as biological indicators of population structure of Halobatrachus didactylus on the Portuguese coast. J Appl Ichthyology 2005b; 21(3): 220224. http://dx.doi.org/10.1111/j.1439-0426.2005.00655.x.

Marques JF, Santos MJ, Gibson DI, Cabral HN, Olson PD. Cryptic species of Didymobothrium rudolphii (Cestoda: Spathebothriidea) from the sand sole, Solea lascaris, off the Portuguese coast, with an analysis of their molecules, morphology, ultrastructure and phylogeny. Parasitology 2007 134(7): 1057-1052. http://dx.doi.org/10.1017/S0031182007002491. PMid:17326848.
Marques JF, Santos MJ, Teixeira CM, Batista MI, Cabral HN. Host-parasite relationships in flatfish (Pleuronectiformes): the relative importance of host biology, ecology and phylogeny. Parasitology 2011; 138(1): 107-121. http://dx.doi.org/10.1017/S0031182010001009. PMid:20819241.

Mattiucci S, Farina V, Campbell N, MacKenzie K, Ramos P, Pinto AL, et al. Anisakis spp. larvae (Nematoda: Anisakidae) from Atlantic horse mackerel: their genetic identification and use as biological tags for host stock characterization. Fish Res 2008; 89(2): 146-151. http:// dx.doi.org/10.1016/j.fishres.2007.09.032.

Mattiucci S, Paggi L, Nascetti G, Portes Santos C, Costa G, Di Beneditto AP, et al. Genetic markers in the study of Anisakis typica (Diesing, 1860): larval identification and genetic relationships with other species of Anisakis Dujardin, 1845 (Nematoda: Anisakidae). Syst Parasitol 2002; 51(3): 159170. http://dx.doi.org/10.1023/A:1014554900808. PMid:11912342.

Meneses I, Ré P. Infection of sardine eggs by a parasitic dinoflagellate Ichthyodinium chabelardi (Hollande et Cachon) of the Portuguese coats. Bol Inst Nac Invest Pescas, Lisboa 1991; 16(2): 1-9.

Menezes J. A case of massive cutaneous myxobolosis in wild mullet. Bol Inst Nac Inv Pescas 1984; 12(2): 71-73.

Menezes J. Hazards from pathogens carried by wild fish particularly wrasse used as lice cleaners. Bull Eur Assoc Fish Pathol 1992; 12(6): 194-195.

Menezes J. Doenças em peixes cultivados no Estuário do Sado e seu controlo. Seminário sobre Recursos Haliêuticos, Ambiente, Aquacultura e Qualidade do Pescado da Península de Setúbal (Setúbal, 26-27 Abril 1994). Publicações Avulsas do IPIMAR 1994; 1: 175-186.

Menezes J. Manual sobre doenças de peixes ósseos. Publicaçôes avulsas do IPIMAR 2000; 3: 213.

Menezes J, Cascalho AFR. Larval paragnathiosis in mullets. Bol Inst Nac Inv Pescas 1984; 12(1): 17-23.

Menezes J, Ramos MA, Pereira TG, Silva AM. Rainbow trout culture failure in a small lake as a result of massive parasitosis related to careless fish introductions. Aquaculture 1990a; 89(2): 123-126. http://dx.doi. org/10.1016/0044-8486(90)90304-6.

Menezes J, Vigário AM, Moledo A, Pita G. Recurso sardinha: parasitose com incidência económica. Pesca e Navegação 1990b; 97(1): 22-24.

Molnár K, Eszterbauer E, Marton S, Székely C, Eiras JC. Comparison of the Myxobolus fauna of common barbel from Hungary and Iberian barbel from Portugal. Dis Aquat Organ 2012; 100(3): 231-248. http:// dx.doi.org/10.3354/dao02469. PMid:22968791.

Moran JDW, Whitaker DJ, Kent ML. A review of the myxosporean genus Kudoa Meglitsch, 1947, and its impact on the international aquaculture industry and commercial fisheries. Aquaculture 1999; 17(1-2): 163-196. http://dx.doi.org/10.1016/S0044-8486(98)00437-2.

Nunes C, Ladeira S, Mergulhão A. Alergia ao Anisakis simplex na população portuguesa. Rev Port Imunoalergol 2003; 11: 30-40.

Oliva ME, Valdivia IM, Costa G, Freitas N, Carvalho MAP, Sánchez $\mathrm{L}$, et al. What can metazoan parasites reveal about the taxonomy of Scomber japonicus Houttuyn in the coast of South America and Madeira Islands? J Fish Biol 2008; 72(3): 545-554. http://dx.doi.org/10.1111/j.10958649.2007.01725.x.

Pereira J, Abrantes C, Martins I, Barata J, Frias P, Pereira I. Ecological and morphological features of Amyloodinium ocellatum occurrences in cultivated gilthead seabream Sparus aurata L.: a case study. Aquaculture 2011; 310(3-4): 289-297. http://dx.doi.org/10.1016/j.aquaculture.2010.11.011. 
Pina S, Barandela S, Santos MJ, Russell-Pinto F, Rodrigues P. Identification and description of Bucephalus minimus (Digenea: Bucephalidae) life cycle in Portugal: morphological, histopathological, and molecular data. $J$ Parasitol 2009; 95(2): 353-359. http://dx.doi.org/10.1645/GE-1719.1. PMid:18710298.

Pinto JS. Parasitic castration in males of Sardina pilchardus (Walb) due to testicular infestation by the coccidia Eimeria sardinae (Thél.). Rev Fac Ciênc Lisboa 1956; 5: 209-224.

Pinto JS, Barraca IF, Assis ME. Nouvelles observations sur la coccidiose par Eimeria sardinae (Thélohan) chez les sardines des environs de Lisbonne, en 1961. Notas Est Inst Biol Marit 1961; 23: 13.

Pontes T, D’Amelio S, Costa G, Paggi L. Molecular characterization of larval anisakid nematodes from marine fishes of Madeira by a PCRbased approach, with evidence for a new species. J Parasitol 2005; 91(6): 1430-1434. http://dx.doi.org/10.1645/GE-565R1.1. PMid:16539027.

Ramos MF, Costa AR, Barandela T, Saraiva A, Rodrigues PN. Scuticociliate infection and pathology in cultured turbot Scophthalmus maximus from the north of Portugal. Dis Aquat Organ 2007; 74(3): 249-253. http:// dx.doi.org/10.3354/dao074249. PMid:17465310.

Ramos P. Anisakis sp.: um risco para a Saúde Pública? Veter Técnica 1998; 3: 30-41.

Ramos P. Anisakis spp. em bacalhau, sushi, e sashimi: risco de infecção parasitária e alergia. Rev Port Cienc Vet 2011; 106(577-580): 87-97.

Rangel LJ, Rocha S, Borkhanuddin MH, Cech G, Castro R, Casal G, et al. Ortholinea aurata n. sp. (Myxozoa, Ortholineidae) infecting the urinary bladder of the gilthead seabream Sparus aurata (Teleostei, Sparidae), in a Portuguese fish farm. Parasitol Res 2014; 113(9): 3427-3437. http:// dx.doi.org/10.1007/s00436-014-4008-4. PMid:24985497.

Rangel LJ, Santos MJ, Cech G, Székely C. Morphology, molecular data, and development of Zschokkella mugilis (Myxosporea, Bivalvulida) in a polychaete alternate host, Nereis diversicolor. J Parasitol 2009; 95(3): 561569. http://dx.doi.org/10.1645/GE-1777.1. PMid:18939897.

Rego AA, Carvalho Varela M, Mendonça MM, Afonso-Roque MM. Helmintofauna da sarda (Scomber scombrus L.) peixe da costa continental Portuguesa. Mem Inst Oswaldo Cruz 1985; 80(1): 97-100. http://dx.doi. org/10.1590/S0074-02761985000100015.

Rocha S, Casal G, Rangel L, Castro R, Severino S, Azevedo C, et al. Ultrastructure and phylogeny of Ceratomyxa auratae n. sp. (Myxosporea: Ceratomyxidae), a parasite infecting the gilthead seabream Sparus aurata (Teleostei: Sparidae). Parasitol Int 2015a; 64(5): 305-313. http://dx.doi. org/10.1016/j.parint.2015.04.002. PMid:25892564.

Rocha S, Casal G, Rangel L, Severino S, Castro R, Santos MJ, et al. Ultrastructure and phylogeny of Ceratomyxa sp. (Myxosporea), a parasite infecting Sparus aurata (Teleostei) in a portuguese fish farm. Microsc Microanal 2015b;21(S5 Suppl 5): 89-90. http://dx.doi.org/10.1017/ S1431927615014257. PMid:26227725.

Rocha S, Casal G, Rangel L, Severino R, Castro R, Azevedo C, et al. Ultrastructural and phylogenetic description of Zschokkella auratis sp. nov. (Myxozoa), a parasite of the gilthead seabream Sparus aurata. Dis Aquat Organ 2013; 107(1): 19-30. http://dx.doi.org/10.3354/dao02661. PMid:24270020.

Rodrigues A, Saraiva A. Spatial distribution \& seasonality of Pseudoctylogyrus anguillae \& P. bini (Monogenea: Pseudodactylogyridae) on the gills of the European eel Anguilla anguilla. Bull Eur Assoc Fish Pathol 1996; 16(3): 85-88.
Rodrigues HO, Carvalho Varela M, Rodrigues SS, Cristófaro R. Alguns trematódeos digenéticos de peixes do Oceano Atlântico - costa continental portuguesa e costa continental da África. Actas Soc Biol Rio de 1972; 15(2): 87-93.

Rodrigues HO, Carvalho Varela M, Rodrigues SS, Cristófaro R. Alguns nematódeos de peixes do Oceano Atlântico: costa continental portuguesa e costa do Norte da África. Mem Inst Oswaldo Cruz 1973; 71(3): 247-256. http://dx.doi.org/10.1590/S0074-02761973000200005.

Rodrigues HO, Carvalho Varela M, Rodrigues SS, Cristófaro R. Notas sobre dois trematódeos digenéticos de peixes do Oceano Atlântico - costa continental portuguesa e costa do Norte de África. Actas Soc Biol Rio de 1975a; 17(3): 121-124.

Rodrigues HO, Carvalho Varela M, Rodrigues SS, Cristófaro R. Nova contribuição para o estudo dos nematódeos de peixes do Oceano Atlântico: costa continental portuguesa e costa do Norte da África. Mem Inst Oswaldo Cruz 1975b; 73(1-2): 127-134. http://dx.doi.org/10.1590/ S0074-02761975000100009.

Rodrigues HO, Noronha D, Carvalho Varela M. Alguns acantocéfalos de peixes do Oceano Atlântico, costa continental portuguesa e do norte da África. Mem Inst Oswaldo Cruz 1975c; 73(3): 209-214. http://dx.doi. org/10.1590/S0074-02761975000200004.

Rombert PC. Anisakiose humana. Acta Parasitol Port 1993; 1(1): 45-47.

Santos MJ. Observations on the parasitofauna of wild sea bass (Dicentrarchus labrax L.) from Portugal. Bull Eur Assoc Fish Pathol 1996; 16(3): 77-79.

Santos MJ, Eiras JC. A seasonal study on the parasitization of Lipophrys pholis (Pisces: Blenniidae) by Helicometra fasciata (Digenea: Opecoelidae) and Lecithochirium furcolabiatum (Digenea: Hemiuridae) in Portugal. Aquaculture 1995; 132(1-2): 175-181. http://dx.doi.org/10.1016/00448486(94)00390-A.

Santos MJ, Gibson DI. Morphological features of Prosorhynchus crucibulum and $P$. aculeatus (Digenea: Bucephalidae), intestinal parasites of Conger conger (Pisces: Congridae), elucidated by scanning electron microscopy. Folia Parasitol (Praha) 2002; 49(2): 96-102. http://dx.doi.org/10.14411/ fp.2002.019. PMid:12194496.

Santos MJ, Saraiva A, Cruz C, Eiras JC, Hermida M, Ventura C, et al. Use of parasites as biological tags in stock identification of black scabbardfish, Aphanopus carbo Lowe, 1839 (Osteichthyes: Trichiuridae) from Portuguese waters. Sci Mar 2009; 73(S2): 55-62. http://dx.doi. org/10.3989/scimar.2009.73s2055.

Saraiva A. Pseudodactylogyrus anguillae (Yin \& Sproston, 1948) Gussev, 1965 and P. bini (Kikuche, 1929) Gussev, 1965 (Monogenea: Monopisthocotylea) in Portugal. Bull Eur Assoc Fish Pathol 1995; 15(3): 81-83.

Saraiva A. Ergasilus gibbus Nordmann, 1832 (Copepoda: Ergasilidae) on the gills of the European eel Anguilla anguilla from Portugal. Res Rev Parasitol 1996; 56(1): 21-24.

Saraiva A, Antão A, Cruz C. Comparative studies of parasite communities in European eel Anguilla anguilla from rivers of northern Portugal. Helminthologia 2005; 42(2): 99-106.

Saraiva A, Chubb JC. Preliminary observations on the parasites of Anguilla anguilla (L.) from Portugal. Bull Eur Assoc Fish Pathol 1989; 9(4): 88-89.

Saraiva A, Costa J, Serrão J, Cruz C, Eiras JC. A histology-based fish health assessment of farmed seabass (Dicentrarchus labrax L.). Aquaculture 2015a; 448: 375-381. http://dx.doi.org/10.1016/j.aquaculture.2015.06.028.

Saraiva A, Costa J, Serrão J, Eiras JC, Cruz C. Study of the gill health status of farmed sea bass (Dicentrarchus labrax L., 1758) using different 
tools. Aquaculture 2015b; 441: 16-20. http://dx.doi.org/10.1016/j. aquaculture.2015.02.004.

Saraiva A, Cruz C, Ferreira S. Studies on Myxidium rhodei Léger, 1905 (Myxozoa: Myxosporea) on Chondrostoma polylepis from River Ave, North Portugal. Bull Eur Assoc Fish Pathol 2000a; 20(3): 106-110.

Saraiva A, Cruz C, Santos MJ, Ferreira S. Nematode parasites from digestive tract of Conger Conger conger L. from the Northwest coast of the Iberian Peninsula. Bull Eur Assoc Fish Pathol 2000b; 20(4): 163-166.

Saraiva A, Eiras JC. Parasite community of european eel Anguilla anguilla (L.) in the river Este, northern Portugal. Res Rev Parasitol 1996; 56(4): 179-183.

Saraiva A, Eiras JC, Cruz C. Fenómenos histopatológicos em peixes provocados por doenças de etiologia parasitária. Acta Parasitol Port 1998; 5(2): 67-72.

Saraiva A, Felisberto P, Cruz C. Occurrence and maturation of Rhabdochona gnedini (Nematoda: Rhabdochonidae) in the barbels of the Sousa river, Portugal. Parasite 2002; 9(1): 81-84. http://dx.doi.org/10.1051/ parasite/200209181. PMid:11938701.

Saraiva A, Hermida M, Costa MJ, Maia C, Reis AR, Cruz C, et al. First record of Philometra ovata (Nematoda) infection in Gobio lozanoi in Portugal. J Fish Biol 2008; 73(9): 2288-2292. http://dx.doi.org/10.1111/j.10958649.2008.02063.x.

Saraiva A, Jerónimo D, Cruz C. Amyloodinium ocellatum (Chromoalveolata: Dinoflagellata) in farmed turbot. Aquaculture 2011; 320(1-2): 34-36. http://dx.doi.org/10.1016/j.aquaculture.2011.07.034.

Saraiva A, Molnár K. Myxobolus portucalensis n. sp. in the fins of the European eel Anguilla anguilla (L.) in Portugal. Rev Iber Parasitol 1990; 50(1-2): 31-35.

Saraiva A, Moravéc F. Redescription of Rhabdochona anguillae (Nematoda: Rhabdochonidae), a parasite of eel, Anguilla anguilla, in Europe. Folia Parasitol (Praha) 1998; 45(3): 233-238. PMid:9805785.

Saraiva A, Moravéc F, Pereira A, Cruz C. Development of Spinitectus inermis (Nematoda: Cystidicolidae), a parasite of eel, Anguilla anguilla, in Europe. Folia Parasitol (Praha) 2002a; 49(2): 118-126. http://dx.doi. org/10.14411/fp.2002.022. PMid:12194484.

Saraiva A, Pereira A, Cruz C. Observations on the occurrence and maturation of Spinitectus inermis (Nematoda: Cystidicolidae) in the Sousa River, Portugal. Folia Parasitol (Praha) 2002b; 49(2): 167-168. http:// dx.doi.org/10.14411/fp.2002.031. PMid:12194492.

Saraiva A, Pereira A, Cruz C. Observations on the occurrence and maturation of Rhabdochona anguillae (Nematoda: Rhabdochonidae) in the Sousa river, Portugal. Helminthologia 2002c; 39(1): 41-43.

Saraiva A, Valente ACN. Black spot disease and Lernaea sp. infestation on Leuciscus cephalus L. (Pisces: Cyprinidae) in Portugal. Bull Eur Assoc Fish Pathol 1988; 8(1): 7-8.

Sarasquete MC, Eiras JC. Hemoprotozoosis en una población de Blennius pholis (L., 1758) de las costas de Oporto (Portugal). Inv Pesq 1985; 49(4): 627-635.

Sequeira V, Gordo LS, Neves A, Paiva RB, Cabral HN, Marques JF. Macroparasites as biological tags for stock identification of the bluemouth,
Helicolenus dactylopterus (Delaroche, 1809) in Portuguese waters. Fish Res 2010; 106(3):321-328. http://dx.doi.org/10.1016/j.fishres.2010.08.014.

Silva A, Miranda A. Laboratory rearing of sardine larvae, Sardina pilchardus (Walb.), and early effects of starvation: a preliminary experiment. Bol Inst Esp Oceanogr 1992; 8(1): 163-174.

Silva MER, Eiras JC. Occurrence of Anisakis sp. in fishes off the Portuguese West Coast and evaluation of its zoonotic potential. Bull Eur Assoc Fish Pathol 2003; 23(1): 13-17.

Skilbrei OT, Finstad B, Urdal K, Bakke G, Kroglund F, Strand R. Impact of early salmon louse, Lepeophtheirus salmonis, infestation and differences in survival and marine growth of sea-ranched Atlantic salmon, Salmo salar L., smolts 1997-2009. J Fish Dis 2013; 36(3): 249-260. http://dx.doi. org/10.1111/jfd.12052. PMid:23311746.

Soares F, Quental Ferreira H, Cunha E, Pousão-Ferreira P. Occurrence of Amyloodinium ocellatum in aquaculture fish production: a serious problem in semi-intensive earthen ponds. Aquacult Eur 2011; 36(4): 13-16.

Stratoudakis Y, Barbosa A, Menezes I. Infection of sardine eggs by the protistan endoparasite Ichthyodinium chabelardi off Portugal. J Fish Biol 2000; 57(2): 476-482. http://dx.doi.org/10.1111/j.1095-8649.2000. tb02186.x.

Świderski Z, Gibson DI, Santos MJ, Poddubnaya LG. Ultrastructure of the intrauterine eggs of Didymobothrium rudolphii (Monticelli, 1890) (Cestoda, Spathebothriidea, Acrobothriidae) and its phylogenetic implications. Acta Parasitol 2010; 55(3): 254-269. http://dx.doi. org/10.2478/s11686-010-0037-z.

Székely C, Eiras JC, Eszterbauer E. Description of a new synactinomyxon type from River Sousa, Portugal. Dis Aquat Organ 2005; 66(1): 9-14. http://dx.doi.org/10.3354/dao066009. PMid:16175962.

Tendeiro J. Sobre um novo digenético, Dolichoenterum manteri sp. n., parasito do safio Conger conger (L.). Algumas consideraçóes sobre a sistemática da ordem Gasterostomata - Odhner, 1905. Bol Soc Port Ciênc Nat 1955; 19(1): 19-43.

Tendeiro J, Valdez V. Helmintologia ictiológica. I. Alguns helmintes do sável, Alosa alosa (L.) das águas portuguesas. Descrição de uma nova espécie, Mazocraes vilelai n. sp. (Trematoda, Monogenea). Bol Cult Guiné Port 1955a; 10(37): 49-80.

Tendeiro J, Valdez V. Helmintologia ictiológica. II. sobre os helmintes de alguns peixes da costa portuguesa. Bol Cult Guiné Port 1955b; 10(37): 81-127.

US Food and Drug Administration. Fish and fisheries products: hazards and controls guidance [online]. 4th ed. Washington, D.C.; 2011 [cited 2016 May 01]. Available from: www.fda.gov/downloads/Food/ GuidanceRegulation/UCM251970.pdf.

Ventura MT, Paperna I. Histopathology of Myxidium giardi Cépède, 1906 infection in European eels, Anguilla anguilla L., in Portugal. Aquaculture 1984; 43(4): 357-368. http://dx.doi.org/10.1016/0044-8486(84)90244-8.

Ventura MT, Paperna I. Histopathology of Ichthyophthirius multifliis infections in fishes. J Fish Biol 1985; 27(2): 185-203. http://dx.doi. org/10.1111/j.1095-8649.1985.tb04020.x.

Worms World Register of Marine Species [online]. 2016 [cited 2016 May 01]. Available from: http://www.marinespecies.org/aphia. php?p=taxdetails\&id=118834 